TRANSACTIONS OF THE

AMERICAN MATHEMATICAL SOCIETY

Volume 356, Number 12, Pages 4737-4766

S 0002-9947(04)03693-1

Article electronically published on June 29, 2004

\title{
COMPLETE SECOND ORDER LINEAR DIFFERENTIAL OPERATOR EQUATIONS IN HILBERT SPACE AND APPLICATIONS IN HYDRODYNAMICS
}

\author{
N. D. KOPACHEVSKY, R. MENNICKEN, JU. S. PASHKOVA, AND C. TRETTER
}

\begin{abstract}
We study the Cauchy problem for a complete second order linear differential operator equation in a Hilbert space $\mathcal{H}$ of the form

$$
\frac{d^{2} u}{d t^{2}}+(F+\mathrm{i} K) \frac{d u}{d t}+B u=f, \quad u(0)=u^{0}, \quad u^{\prime}(0)=u^{1} .
$$

Problems of this kind arise, e.g., in hydrodynamics where the coefficients $F$, $K$, and $B$ are unbounded selfadjoint operators. It is assumed that $F$ is the dominating operator in the Cauchy problem above, i.e.,

$$
\mathcal{D}(F) \subset \mathcal{D}(B), \quad \mathcal{D}(F) \subset \mathcal{D}(K) .
$$

We also suppose that $F$ and $B$ are bounded from below, but the operator coefficients are not assumed to commute. The main results concern the existence of strong solutions to the stated Cauchy problem and applications of these results to the Cauchy problem associated with small motions of some hydrodynamical systems.
\end{abstract}

\section{INTRODUCTION}

Cauchy problems of the form

$$
\frac{d^{2} u}{d t^{2}}+(F+\mathrm{i} K) \frac{d u}{d t}+B u=f, \quad u(0)=u^{0}, \quad u^{\prime}(0)=u^{1},
$$

arise for example in the study of small motions of hydrodynamical systems. There $u$ represents the displacement of the given system from its equilibrium and $f$ is a small field of external forces. The operator coefficients in (1.1) and the assumptions on them have direct physical meaning. The operator $F$ is the operator of energy dissipation and therefore $F \geq 0$. In some special cases, e.g., for the Navier-Stokes equations describing the motion of a viscous fluid, $F$ is even a strongly positive operator. The operator $K$ is the Coriolis operator, which takes into account the influence of Coriolis forces when the system rotates near some fixed axis with constant angular velocity. Since the Coriolis forces do not generate work, $K$ is usually a bounded selfadjoint operator. The operator $B$ represents the potential energy

Received by the editors January 29, 2002.

2000 Mathematics Subject Classification. Primary 35A05; Secondary 35Q30, 47D06, 47F05, 47B44, 47B50.

Key words and phrases. Block operator matrix, differential equation in Hilbert space, evolution problem, Navier-Stokes equations.

N. D. Kopachevsky, R. Mennicken, and C. Tretter gratefully acknowledge the support of the German Research Foundation, DFG, Grants No. 436 UKR 113/38/0 and No. TR368/4-1, and of the British Engineering and Physical Sciences Research Council, EPSRC, Grant No. GR/R40753. 
operator and is thus selfadjoint and in general unbounded. If the system under consideration is statically stable in linear approximation, then $B$ is nonnegative. The role of the operator of kinetic energy is played by the identity operator $I$ (as the coefficient of the second derivative in (1.1)).

Abstract Cauchy problems (1.1) for complete second order linear differential operator equations in a Hilbert space $\mathcal{H}$ have been studied since the sixties using two approaches. The first one uses the theory of semigroups of linear operators acting in Banach or Hilbert spaces. Here we only mention the classical results of E. Hille and R.S. Phillips $[\mathrm{HP}]$ and subsequent investigations of S. G. Krein [K2 (see also the monographs of M. A. Krasnoselsky, P. P. Zabrejko, E. I. Pustyl'nik, and P. E. Sobolevsky [KZPP], S. G. Krein and M. I. Hazan [KH], D. Goldstein [Go, and the bibliographies therein).

The second approach is based on the application of so-called operator cosinefunctions for incomplete linear differential operator equations of second order. Here we refer to the papers of M. Sowa [S1, S2], and to the monographs of H. O. Fattorini [F] and V. I. Gorbachuk and M. L. Gorbachuk [GG]. Complete differential operator equations in Banach spaces with commuting operator coefficients were investigated by V. K. Ivanov, A. I. Mel'nikova, and A. I. Filinkov [IMF].

The new approach used in the present paper is to transform the second order linear differential operator equation (1.1) into a system of two first order linear differential operator equations. This system can be written as a first order linear differential operator equation where the coefficients are $2 \times 2$ block operator matrices in a product of Hilbert spaces $\mathcal{H} \oplus \mathcal{H}$ with unbounded entries, which are in general not closed even when the entries are closed.

The theory of block operator matrices with unbounded entries has been a vital area of research over the last decade, motivated by applications from hydrodynamics, magnetohydrodynamics, and quantum mechanics. Contributions to this field are due to R. Nagel [N1, N2, N3], K.-J. Engel [En1, En2, F. V. Atkinson, H. Langer, R. Mennicken, A. A. Shkalikov ALMS, V. M. Adamyan, H. Langer AdL, A. Motovilov [M], V. M. Adamyan, H. Langer, R. Mennicken, J. Saurer AdLMS, R. Mennicken, A. A. Shkalikov [MS], A. A. Shkalikov [S], A. Yu. Konstantinov Kon], T. Ya. Azizov, N. D. Kopachevsky, L. D. Orlova [AKO], H. Langer, C. Tretter [LT1 LT2], R. Mennicken, A. Motovilov [MM], V.Hardt, R. Mennicken, S. Naboko [HMN], R. Mennicken, S. Naboko, C. Tretter [MNT], T. Ya. Azizov, V. Hardt, N. D. Kopachevsky, R. Mennicken [AHKM], and others. In particular, it has been shown that under certain assumptions on the domains of the entries a block operator matrix has a closure, and in applications, e.g., from hydrodynamics or magnetohydrodynamics this closure corresponds to the underlying initial boundary value problem in a natural way.

By means of these block operator techniques we solve the problem of wellposedness of the Cauchy problem (1.1) with operator coefficients $F$ and $B$ which are bounded from below. In Section 2, we show that problem (1.1) can be reduced to a Cauchy problem

$$
\frac{d y}{d t}+\mathcal{A} y=\hat{f}, \quad y(0)=y^{0},
$$

in the Hilbert space $\mathcal{H}^{2}:=\mathcal{H} \oplus \mathcal{H}$ where $\mathcal{A}$ is a closed block operator matrix. To this end, we first consider the case $F \gg 0, B \geq 0$ and $K$ bounded, then the case $F, B$ semibounded and $K$ bounded, and, finally, the most general case $F, B$ semibounded 
and $K$ unbounded. It turns out that in each case the constructed operator $\mathcal{A}$ is accretive in $\mathcal{H}^{2}$. This property enables us to investigate problem (1.2) using the theory of contractive semigroups (with $-\mathcal{A}$ as a generator). In Section 3 , we apply our results to S. G. Krein's problem of small motions of a viscous fluid in an arbitrary open vessel [K1], [KKN], [KL], AKL], Ga], [Ko].

\section{The EVOlution PRoblem}

2.1. Statement of the evolution problem. In a separable Hilbert space $\mathcal{H}$ we consider a Cauchy problem for a complete second order linear differential operator equation of the form

$$
\frac{d^{2} u}{d t^{2}}+(F+1 K) \frac{d u}{d t}+B u=f, \quad u(0)=u^{0}, \quad u^{\prime}(0)=u^{1} .
$$

Here the coefficients $F, K$, and $B$ are linear operators acting in $\mathcal{H}$ such that

$$
\begin{aligned}
& F=F^{*} \geq \gamma_{F} I \text { for some } \gamma_{F} \in \mathbb{R}, \\
& K=K^{*}, \quad \mathcal{D}(F) \subset \mathcal{D}(K), \\
& B=B^{*} \geq \gamma_{B} I \text { for some } \gamma_{B} \in \mathbb{R}, \quad \mathcal{D}(F) \subset \mathcal{D}(B),
\end{aligned}
$$

$f$ is a given function of $t$ with values in $\mathcal{H}, u^{0}, u^{1} \in \mathcal{H}$ are given initial values, and $u$ is an unknown function of $t$ with values in $\mathcal{H}$.

Definition 2.1. Let $T>0$. A function $u$ is called a strong solution of the Cauchy problem (2.1) on the interval $[0, T]$ if $u(t) \in \mathcal{D}(B), d u(t) / d t \in \mathcal{D}(F)$ for all $t \in[0, T]$, the functions $B u, F d u / d t$, and $d^{2} u / d t^{2}$ belong to the space $C[0, T ; \mathcal{H}]$, and $u$ satisfies (2.1) on $[0, T]$.

Here and in the following, $C^{k}[0, T ; \mathcal{H}], k \in \mathbb{N}_{0}$, denotes the space of all $k$ times continuously differentiable functions on $[0, T]$ with values in $\mathcal{H}$, and we write $C[0, T ; \mathcal{H}]$ for $C^{0}[0, T ; \mathcal{H}]$.

It follows from the definition that for a strong solution $u$ of problem (2.1) we have

$$
u(0)=u^{0} \in \mathcal{D}(B), \quad u^{\prime}(0)=u^{1} \in \mathcal{D}(F) .
$$

2.2. Transformation to a Cauchy problem for a first order differential equation with block operator matrix coefficients. We begin by considering the special case

$$
F \gg 0, \quad B \geq 0, \quad K \in \mathcal{L}(\mathcal{H})
$$

where $\mathcal{L}(\mathcal{H})$ denotes the space of bounded linear operators in $\mathcal{H}$.

Let the function $u$ be a strong solution of problem (2.1) on some interval $[0, T]$. Then the function $v$ given by

$$
\frac{d v}{d t}=-{ }_{1} B^{1 / 2} u, \quad v(0)=0
$$

belongs to $C^{2}[0, T ; \mathcal{H}]$ and

$$
\frac{d^{2} v}{d t^{2}}=-{ }_{1} B^{1 / 2} \frac{d u}{d t}, \quad v^{\prime}(0)=-1 B^{1 / 2} u^{0}
$$


Hence, with (2.7), (2.8), the Cauchy problem (2.1) can be written as a Cauchy problem of the form

$$
\begin{aligned}
& \frac{d^{2}}{d t^{2}}\left(\begin{array}{l}
u \\
v
\end{array}\right)+\left(\begin{array}{cc}
F+1 K & 1 B^{1 / 2} \\
1 B^{1 / 2} & 0
\end{array}\right) \frac{d}{d t}\left(\begin{array}{l}
u \\
v
\end{array}\right)=\left(\begin{array}{l}
f \\
0
\end{array}\right), \\
& u(0)=u^{0}, \quad u^{\prime}(0)=u^{1}, \quad v(0)=0, \quad v^{\prime}(0)=-1 B^{1 / 2} u^{0} .
\end{aligned}
$$

Define the function $y$ of $t$ with values in $\mathcal{H}^{2}:=\mathcal{H} \oplus \mathcal{H}$ by

$$
y=\left(\frac{d u}{d t}, \frac{d v}{d t}\right)^{\mathrm{t}}
$$

where ${ }^{\mathrm{t}}$ denotes the transpose, and let $\mathcal{A}_{0}$ be the block operator matrix given by

$$
\mathcal{A}_{0}:=\left(\begin{array}{cc}
F+1 K & 1 B^{1 / 2} \\
1 B^{1 / 2} & 0
\end{array}\right)
$$

with domain

$$
\mathcal{D}\left(\mathcal{A}_{0}\right):=\mathcal{D}(F) \oplus \mathcal{D}\left(B^{1 / 2}\right) .
$$

Then, if $(u, v)^{\mathrm{t}}$ is a solution of problem (2.9), (2.10), the function $y$ is a solution of

$$
\frac{d y}{d t}+\mathcal{A}_{0} y=\hat{f}_{0}, \quad y(0)=y^{0}
$$

where

$$
\hat{f}_{0}:=\left(\begin{array}{l}
f \\
0
\end{array}\right), \quad y^{0}:=\left(\begin{array}{c}
u^{1} \\
-1 B^{1 / 2} u^{0}
\end{array}\right)
$$

i.e., of a Cauchy problem for a first order differential equation in $\mathcal{H}^{2}=\mathcal{H} \oplus \mathcal{H}$.

Vice versa, if $y=\left(y_{1}, y_{2}\right)^{\mathrm{t}}$ is a solution of (2.14), (2.15), then the functions $u, v$ given by

$$
u(t):=\int_{0}^{t} y_{1}(\tau) d \tau+u^{0}, \quad v(t):=\int_{0}^{t} y_{2}(\tau) d \tau, \quad t \in[0, T]
$$

are solutions of (2.9), (2.10).

Lemma 2.2. The block operator matrix $\mathcal{A}_{0}$ given by (2.12) with domain (2.13) is an accretive operator in $\mathcal{H}^{2}$, i.e.,

$$
\operatorname{Re}\left(\mathcal{A}_{0} y, y\right)_{\mathcal{H}^{2}} \geq 0, \quad y \in \mathcal{D}\left(\mathcal{A}_{0}\right) .
$$

Proof. For $y=\left(y_{1}, y_{2}\right)^{\mathrm{t}} \in \mathcal{D}\left(\mathcal{A}_{0}\right)$ we have $\operatorname{Re}\left(\mathcal{A}_{0} y, y\right)_{\mathcal{H}^{2}}=\left(F y_{1}, y_{1}\right)_{\mathcal{H}} \geq 0$ by the assumption (2.6) on $F$.

Now let $a>0$ and introduce a new unknown function $z$ by the relation

$$
y(t)=\mathrm{e}^{a t} z(t), \quad t \in[0, T] .
$$

Then, by (2.14), (2.15), $z$ is a solution of the Cauchy problem

$$
\frac{d z}{d t}+\mathcal{A}_{a} z=\hat{f}_{a}, \quad z(0)=y^{0}
$$

where

$$
\hat{f}_{a}(t):=\mathrm{e}^{-a t} \hat{f}_{0}(t)=\mathrm{e}^{-a t}\left(\begin{array}{c}
f(t) \\
0
\end{array}\right), \quad t \in[0, T]
$$


and

$$
\mathcal{A}_{a}:=\mathcal{A}_{0}+a \mathcal{I}=\left(\begin{array}{cc}
F_{a}+1 K & 1 B^{1 / 2} \\
1 B^{1 / 2} & a I
\end{array}\right), \quad F_{a}:=F+a I,
$$

where $\mathcal{I}$ and $I$ are the identity operators in $\mathcal{H}^{2}$ and $\mathcal{H}$, respectively. It follows from Lemma 2.2 that the operator $\mathcal{A}_{a}$ is uniformly accretive:

$$
\operatorname{Re}\left(\mathcal{A}_{a} z, z\right)_{\mathcal{H}^{2}} \geq a\|z\|_{\mathcal{H}^{2}}^{2}, \quad z \in \mathcal{D}\left(\mathcal{A}_{a}\right)=\mathcal{D}\left(\mathcal{A}_{0}\right) .
$$

2.3. Transformation to a differential equation with maximal uniformly accretive operator coefficient. If the operator $B \geq 0$ is bounded, then the operator $\mathcal{A}_{a}$ is closed and maximal uniformly accretive on its domain $\mathcal{D}\left(\mathcal{A}_{a}\right)=$ $\mathcal{D}(F) \oplus \mathcal{H}$. However, this need not be true for unbounded $B$ in general. In the following we shall prove that the closure of the operator $\mathcal{A}_{a}$ is maximal uniformly accretive.

We introduce the following operators:

$$
Q_{a}:=B^{1 / 2} F_{a}^{-1 / 2}, \quad Q_{a}^{+}:=F_{a}^{-1 / 2} B^{1 / 2}, \quad \mathcal{D}\left(Q_{a}^{+}\right)=\mathcal{D}\left(B^{1 / 2}\right) .
$$

Lemma 2.3. The operator $Q_{a}$ is bounded, and for $Q_{a}^{+}$we have

$$
Q_{a}^{+}=Q_{a}^{*} \mid \mathcal{D}\left(B^{1 / 2}\right), \quad \overline{Q_{a}^{+}}=Q_{a}^{*} \in \mathcal{L}(\mathcal{H}) .
$$

Proof. First we show that $Q_{a} \in \mathcal{L}(\mathcal{H})$. Indeed, by Heinz' inequality (see, e.g., EE Chapter III, Proposition 8.12]), the inclusion $\mathcal{D}(B) \supset \mathcal{D}(F)$ (see (2.4)) implies that $\mathcal{D}\left(B^{1 / 2}\right) \supset \mathcal{D}\left(F^{1 / 2}\right)=\mathcal{D}\left(F_{a}^{1 / 2}\right)$, and therefore $Q_{a}$ is defined everywhere. But $Q_{a}$ is closed and hence $Q_{a} \in \mathcal{L}(\mathcal{H})$ and also $Q_{a}^{*} \in \mathcal{L}(\mathcal{H})$.

Since $Q_{a}^{+}=\left(F_{a}^{-1 / 2}\right)^{*}\left(B^{1 / 2}\right)^{*} \subset\left(B^{1 / 2} F_{a}^{-1 / 2}\right)^{*}=Q_{a}^{*}$, the operator $Q_{a}^{+}$is bounded on $\mathcal{D}\left(B^{1 / 2}\right)$ and $Q_{a}^{+}=Q_{a}^{*} \mid \mathcal{D}\left(B^{1 / 2}\right)$. Since $\mathcal{D}\left(B^{1 / 2}\right)$ is dense in $\mathcal{H}$, we obtain $\overline{Q_{a}^{+}}=$ $Q_{a}^{*}$.

Theorem 2.4. The block operator matrix $\mathcal{A}_{a}$ defined in (2.18) has the following two representations on $\mathcal{D}(F) \oplus \mathcal{D}\left(B^{1 / 2}\right)$ :

i) in Schur-Frobenius form:

$$
\mathcal{A}_{a}=\left(\begin{array}{cc}
I & 0 \\
{ }_{1} Q_{a} F_{a}^{-1 / 2} & I
\end{array}\right)\left(\begin{array}{cc}
F_{a} & 0 \\
0 & a I+Q_{a} Q_{a}^{+}
\end{array}\right)\left(\begin{array}{cc}
I & 1 F_{a}^{-1 / 2} Q_{a}^{+} \\
0 & I
\end{array}\right)+\left(\begin{array}{cc}
1 K & 0 \\
0 & 0
\end{array}\right),
$$

ii) with symmetric outer factors:

$$
\mathcal{A}_{a}=\left(\begin{array}{cc}
F_{a}^{1 / 2} & 0 \\
0 & I
\end{array}\right)\left(\begin{array}{cc}
I+{ }_{1} F_{a}^{-1 / 2} K F_{a}^{-1 / 2} & 1 Q_{a}^{+} \\
1 Q_{a} & a I
\end{array}\right)\left(\begin{array}{cc}
F_{a}^{1 / 2} & 0 \\
0 & I
\end{array}\right) .
$$

The operator $\mathcal{A}_{a}$ is closable, its closure $\mathcal{A}:=\overline{\mathcal{A}_{a}}$ is maximal uniformly accretive with

$$
\operatorname{Re}(\mathcal{A} z, z)_{\mathcal{H}^{2}} \geq a\|z\|_{\mathcal{H}^{2}}^{2}, \quad z \in \mathcal{D}(\mathcal{A})
$$

and it admits the following two representations:

i) in Schur-Frobenius form:

$$
\mathcal{A}=\left(\begin{array}{cc}
I & 0 \\
{ }_{1} Q_{a} F_{a}^{-1 / 2} & I
\end{array}\right)\left(\begin{array}{cc}
F_{a} & 0 \\
0 & a I+Q_{a} Q_{a}^{*}
\end{array}\right)\left(\begin{array}{cc}
I & { }_{1} F_{a}^{-1 / 2} Q_{a}^{*} \\
0 & I
\end{array}\right)+\left(\begin{array}{cc}
1 K & 0 \\
0 & 0
\end{array}\right)
$$


ii) with symmetric outer factors:

$$
\mathcal{A}=\left(\begin{array}{cc}
F_{a}^{1 / 2} & 0 \\
0 & I
\end{array}\right)\left(\begin{array}{cc}
I+1 F_{a}^{-1 / 2} K F_{a}^{-1 / 2} & 1 Q_{a}^{*} \\
1 Q_{a} & a I
\end{array}\right)\left(\begin{array}{cc}
F_{a}^{1 / 2} & 0 \\
0 & I
\end{array}\right) .
$$

The operator $\mathcal{A}$ has the domain

$$
\mathcal{D}(\mathcal{A})=\left\{z=\left(z_{1}, z_{2}\right)^{\mathrm{t}} \in \mathcal{H}^{2}: z_{1}+{ }_{1} F_{a}^{-1 / 2} Q_{a}^{*} z_{2} \in \mathcal{D}\left(F_{a}\right)\right\}
$$

and, for $z \in \mathcal{D}(\mathcal{A})$, we have

$$
\mathcal{A} z=\left(\begin{array}{c}
F_{a}\left(z_{1}+{ }_{1} F_{a}^{-1 / 2} Q_{a}^{*} z_{2}\right)+{ }_{1} K z_{1} \\
1 Q_{a} F_{a}^{1 / 2} z_{1}+a z_{2}
\end{array}\right)=\left(\begin{array}{c}
F_{a}^{1 / 2}\left(F_{a}^{1 / 2} z_{1}+1 Q_{a}^{*} z_{2}\right)+{ }_{1} K z_{1} \\
1 Q_{a} F_{a}^{1 / 2} z_{1}+a z_{2}
\end{array}\right) .
$$

Proof. The formulas (2.21) and (2.22) can be checked directly for elements from $\mathcal{D}\left(\mathcal{A}_{a}\right)=\mathcal{D}\left(\mathcal{A}_{0}\right)=\mathcal{D}(F) \oplus \mathcal{D}\left(B^{1 / 2}\right)$. Denote the three factors in the products on the right-hand sides of (2.21) and (2.22) by $R, S$, and $T$.

In the product on the right side of (2.21), the first factor $R$ is everywhere defined and bounded with bounded inverse and the third factor $T$ is densely defined and bounded with closure $\bar{T}$ which has a bounded inverse. The middle factor $S$ is closable with $\mathcal{D}(S) \subset R(T)$ and its closure, which is obtained by replacing $Q_{a}^{+}$by $Q_{a}^{*}$, is strictly positive which shows that its range is all of the space $\mathcal{H} \oplus \mathcal{H}$. Therefore the product $R S T$ is closable and its closure, which is obtained by taking the closures of $S$ and $T$, has range $\mathcal{H} \oplus \mathcal{H}$. But the closure of $R S T$ is also accretive (and hence maximal accretive) since $\mathcal{A}$ is accretive by $(2.19)$ and $K=K^{*}$. Altogether, the closure of RST in (2.21) is maximal uniformly accretive and the second term on the right-hand side of (2.21) is bounded and its real part is identically 0 since $K=K^{*}$. This implies that $\mathcal{A}=\overline{\mathcal{A}_{a}}$ as the sum of the latter is maximal uniformly accretive (see [EE, Chapter III, Corollary 8.5]), satisfies estimate (2.23) and has the representation (2.24). From (2.24), it is easy to see that the domain of $\mathcal{A}$ is given by (2.26) and that the action of $\mathcal{A}$ is determined by (2.27).

The first factor $R$ and the third factor $T$ in the product on the right side of (2.22) are closed and boundedly invertible, while the middle factor $S$ is bounded and densely defined. For the closure of $S$, which is obtained from $S$ by replacing $Q_{a}^{+}$by $Q_{a}^{*}$, we have

$\operatorname{Re}\left(\left(\begin{array}{cc}I+{ }_{1} F_{a}^{-1 / 2} K F_{a}^{-1 / 2} & 1 Q_{a}^{*} \\ 1 Q_{a} & a I\end{array}\right)\left(\begin{array}{c}z_{1} \\ z_{2}\end{array}\right),\left(\begin{array}{c}z_{1} \\ z_{2}\end{array}\right)\right)=\left\|z_{1}\right\|^{2}+a\left\|z_{2}\right\|^{2} \geq \min \{1, a\}\|z\|_{\mathcal{H}^{2}}^{2}$,

that is, $\bar{S}$ is uniformly accretive and hence maximal uniformly accretive because it is bounded. In particular, $\bar{S}$ is also boundedly invertible and thus $\bar{S} T$ is closed which, in turn, implies that $R \bar{S} T$ is closed. Hence $R \bar{S} T$ is a closed extension of $\mathcal{A}_{a}$ and so $\mathcal{A}=\overline{\mathcal{A}_{a}} \subset R \bar{S} T$. It is not difficult to see that the domain of $R \bar{S} T$ is given by

$$
\mathcal{D}(R \bar{S} T)=\left\{z=\left(z_{1}, z_{2}\right)^{\mathrm{t}} \in \mathcal{D}\left(F_{a}^{1 / 2}\right) \oplus \mathcal{H}: F_{a}^{1 / 2} z_{1}+{ }_{1} Q_{a}^{*} z_{2} \in \mathcal{D}\left(F_{a}^{1 / 2}\right)\right\}
$$

which is obviously contained in the domain of $\mathcal{A}$ given by (2.26). Hence $\mathcal{A}=R \bar{S} T$, which proves the second representation in 2.25).

Corollary 2.5. The operator $-\mathcal{A}$ is the generator of the contractive semigroup

$$
\mathcal{U}(t)=\exp (-t \mathcal{A}), \quad\|\mathcal{U}(t)\| \leq \mathrm{e}^{-a t}, \quad t \geq 0 .
$$


Proof. The statements are immediate from the facts that $\mathcal{A}$ is maximal uniformly accretive by Theorem 2.4 and that inequality (2.23) holds (see [Ka Chapter IX]).

2.4. Theorem on well-posedness. The properties of the operator $\mathcal{A}$ proved in the previous subsection allow us to make use of the following known fact (see [K2. Theorem 6.5, Section 1.6.2], or [KZPP, $\overline{\mathrm{KH}}$, Go]).

Theorem 2.6. Let the conditions

$$
y^{0} \in \mathcal{D}(\mathcal{A}), \quad \hat{f}_{a} \in C^{1}\left[0, T ; \mathcal{H}^{2}\right]
$$

hold. Then the Cauchy problem

$$
\frac{d z}{d t}+\mathcal{A} z=\hat{f}_{a}, \quad z(0)=z^{0}=y^{0}
$$

has the unique strong solution

$$
z(t)=\mathcal{U}(t) y^{0}+\int_{0}^{t} \mathcal{U}(t-s) \hat{f}_{a}(s) d s
$$

where $\mathcal{U}(t)$ is the semigroup generated by $\mathcal{A}$ as in (2.28).

Here we recall that $z$ is said to be a strong solution of a Cauchy problem of the form (2.30) on $[0, T]$ if $z(t) \in \mathcal{D}(\mathcal{A})$ for all $t \in[0, T], \mathcal{A} z \in C\left[0, T ; \mathcal{H}^{2}\right], z \in$ $C^{1}\left[0, T ; \mathcal{H}^{2}\right]$, and (2.30) holds on the interval $[0, T]$ (see, e.g., [K2]).

Theorem 2.7. Suppose that the Cauchy problem (2.1) satisfies the conditions

If

$$
F \gg 0, \quad B \geq 0, \quad \mathcal{D}(F) \subset \mathcal{D}(B), \quad K=K^{*} \in \mathcal{L}(\mathcal{H}) .
$$

then the Cauchy problem (2.1) has a unique strong solution on the segment $[0, T]$.

Proof. Step 1. By definition (see (2.17)), $\hat{f}_{a}(t):=\mathrm{e}^{-a t}(f(t), 0)^{\mathrm{t}}, t \in[0, T]$, and hence it follows from (2.32) that

$$
\hat{f}_{a} \in C^{1}\left[0, T ; \mathcal{H}^{2}\right] .
$$

Further, again by assumption (2.32),$u^{1} \in \mathcal{D}(F)$ and $-1 B^{1 / 2} u^{0} \in \mathcal{D}\left(B^{1 / 2}\right)$ and therefore, by (2.15), (2.13),

$$
y^{0}=y(0)=\left(\begin{array}{c}
u^{1} \\
\left.-1 B^{1 / 2} u^{0}\right)
\end{array}\right) \in \mathcal{D}(F) \oplus \mathcal{D}\left(B^{1 / 2}\right)=\mathcal{D}\left(\mathcal{A}_{0}\right)=\mathcal{D}\left(\mathcal{A}_{a}\right) \subset \mathcal{D}(\mathcal{A}) .
$$

Step 2. Consider now the Cauchy problem $(2.30)$ with the operator $\mathcal{A}$ from (2.27) (see Theorem 2.4). It follows from (2.33) and (2.34) that the conditions in (2.29) hold. Thus, by Theorem 2.6 the problem (2.30) has a unique strong solution $z=\left(z_{1}, z_{2}\right)^{\mathrm{t}}$ on $[0, T]$. By (2.27) and (2.17), this means that for all $t \in[0, T]$ the equations

$$
\begin{aligned}
& \frac{d z_{1}}{d t}(t)+F_{a}\left(z_{1}(t)+{ }_{1} F_{a}^{-1 / 2} Q_{a}^{*} z_{2}(t)\right)+1 K z_{1}(t)=\mathrm{e}^{-a t} f(t), \\
& \frac{d z_{2}}{d t}(t)+a z_{2}(t)+{ }_{1} Q_{a} F_{a}^{1 / 2} z_{1}(t)=0
\end{aligned}
$$

and the initial conditions

$$
z_{1}(0)=u^{1}, \quad z_{2}(0)=-1 B^{1 / 2} u^{0}
$$


hold. In (2.35), (2.36) all terms are continuous functions of $t$ with values in the space $\mathcal{H}$ and, by (2.26) , we have $z_{1}(t)+{ }_{1} F_{a}^{-1 / 2} Q_{a}^{*} z_{2}(t) \in \mathcal{D}\left(F_{a}\right)=\mathcal{D}(F)$ for all $t \in[0, T]$.

From (2.36) and the second condition in (2.37) we conclude

$$
z_{2}(t)=-1 \mathrm{e}^{-a t} B^{1 / 2} u^{0}-1 \int_{0}^{t} \mathrm{e}^{-a(t-s)} Q_{a} F_{a}^{1 / 2} z_{1}(s) d s, \quad t \in[0, T] .
$$

Substituting (2.38) into (2.35), we obtain that the function $z_{1}$ is a solution of the Cauchy problem

$$
\begin{aligned}
& \frac{d z_{1}}{d t}(t)+F_{a}\left(z_{1}(t)+F_{a}^{-1 / 2} Q_{a}^{*}\left(\mathrm{e}^{-a t} B^{1 / 2} u^{0}+\int_{0}^{t} \mathrm{e}^{-a(t-s)} Q_{a} F_{a}^{1 / 2} z_{1}(s) d s\right)\right) \\
& +{ }_{1} K z_{1}(t)=\mathrm{e}^{-a t} f(t), \quad z_{1}(0)=u^{1},
\end{aligned}
$$

and hence $z_{1} \in C^{1}[0, T ; \mathcal{H}]$. Here, for all $t \in[0, T]$,

$$
\varphi(t):=z_{1}(t)+\mathrm{e}^{-a t} F_{a}^{-1 / 2} Q_{a}^{*} B^{1 / 2} u^{0}+F_{a}^{-1 / 2} Q_{a}^{*} \int_{0}^{t} \mathrm{e}^{-a(t-s)} Q_{a} F_{a}^{1 / 2} z_{1}(s) d s
$$

belongs to $\mathcal{D}\left(F_{a}\right)$ and $F_{a} \varphi \in C[0, T ; \mathcal{H}]$. Moreover, the function $\varphi_{0}$ given by

$$
\varphi_{0}(t):=\mathrm{e}^{-a t} F_{a}^{-1 / 2} Q_{a}^{*} B^{1 / 2} u^{0}, \quad t \in[0, T],
$$

also has these properties. Indeed, since $u^{0} \in \mathcal{D}(B)$, we have $B^{1 / 2} u^{0} \in \mathcal{D}\left(B^{1 / 2}\right)$, and, by Lemma 2.3

$$
\varphi_{0}(t)=\mathrm{e}^{-a t} F_{a}^{-1 / 2} Q_{a}^{+} B^{1 / 2} u^{0}=\mathrm{e}^{-a t} F_{a}^{-1} B u^{0} \in \mathcal{D}\left(F_{a}\right), \quad t \in[0, T] .
$$

Step 3. We rewrite relation (2.40) in the form

$$
z_{1}(t)+\int_{0}^{t} \mathrm{e}^{-a(t-s)} F_{a}^{-1 / 2} Q_{a}^{*} Q_{a} F_{a}^{1 / 2} z_{1}(s) d s=\varphi(t)-\varphi_{0}(t)=: \varphi_{1}(t) .
$$

By what has been shown above, we have $\varphi_{1}(t) \in \mathcal{D}\left(F_{a}\right)$ and $F_{a} \varphi_{1} \in C[0, T ; \mathcal{H}]$. We introduce the Hilbert space $\mathcal{H}_{F_{a}}=\left(\mathcal{D}\left(F_{a}\right),\|\cdot\|_{F_{a}}\right)$ with the inner product induced by the positive operator $F_{a}$, i.e., the norm being given by

$$
\|u\|_{F_{a}}=\left\|F_{a} u\right\|, \quad u \in \mathcal{D}\left(F_{a}\right)
$$

Since $\mathcal{D}(B) \supset \mathcal{D}(F)=\mathcal{D}\left(F_{a}\right)$, the definition of $Q_{a}$ and $Q_{a}^{+}$in (2.20) and Lemma 2.3 imply that for all $u \in \mathcal{D}\left(F_{a}\right)$,

$$
T u:=F_{a}^{-1 / 2} Q_{a}^{*} Q_{a} F_{a}^{1 / 2} u=F_{a}^{-1 / 2} Q_{a}^{+} B^{1 / 2} u=F_{a}^{-1} B u \in \mathcal{D}\left(F_{a}\right) .
$$

Hence $\left.T\right|_{\mathcal{D}\left(F_{a}\right)}$ is a bounded operator acting in the Hilbert space $\mathcal{H}_{F_{a}}$.

Equation (2.43) can be considered as an integral Volterra equation of the second kind for $z_{1}$ in the space $C\left[0, T ; \mathcal{H}_{F_{a}}\right]$. The function $\varphi_{1} \in C\left[0, T ; \mathcal{H}_{F_{a}}\right]$ on the right-hand side is considered to be given and the kernel $\mathrm{e}^{-a(t-s)} F_{a}^{-1 / 2} Q_{a}^{*} Q_{a} F_{a}^{1 / 2}=$ $\mathrm{e}^{-a(t-s)} F_{a}^{-1} B$ is an operator function with values in $\mathcal{H}_{F_{a}}$ which is continuous in $t$ and $s$. By means of the method of successive approximations (see, e.g., [KKMRSZ, Theorem 1.8]), it can be shown that equation (2.43) has a unique solution $z_{1} \in C\left[0, T ; \mathcal{H}_{F_{a}}\right]$, and therefore each term in equation (2.43) belongs to the space $C\left[0, T ; \mathcal{H}_{F_{a}}\right]$. 
Step 4 . Since $z_{1} \in C\left[0, T ; \mathcal{D}\left(F_{a}\right)\right]$ by Step 3 , we can open the brackets in equation (2.39) which yields

$$
\frac{d z_{1}}{d t}(t)+F_{a} z_{1}(t)+{ }_{1} K z_{1}(t)+\int_{0}^{t} \mathrm{e}^{-a(t-s)} B z_{1}(s) d s+B u^{0}=\mathrm{e}^{-a t} f(t) .
$$

By (2.16) and 2.11) we have

$$
z_{1}(t)=\mathrm{e}^{-a t} y_{1}(t)=\mathrm{e}^{-a t} u^{\prime}(t), \quad t \in[0, T]
$$

Substituting this into equation (2.45), we obtain, after multiplication by $\mathrm{e}^{a t}$,

$$
\frac{d^{2} u}{d t^{2}}+\left(F_{a}-a I+{ }_{1} K\right) \frac{d u}{d t}+B u=f
$$

i.e., equation (2.1). In this equation all terms belong to the space $C[0, T ; \mathcal{H}]$, which means that $u$ is a strong solution of (2.1).

In the next stage, we consider the case when the conditions $F \gg 0$ and $B \geq 0$ no longer hold, but $K$ is still bounded.

Theorem 2.8. Suppose that there are real constants $\gamma_{F}$ and $\gamma_{B}$ such that

$$
F \geq \gamma_{F} I, \quad B \geq \gamma_{B} I, \quad \mathcal{D}(F) \subset \mathcal{D}(B), \quad K=K^{*} \in \mathcal{L}(\mathcal{H}),
$$

and assume

$$
u^{0} \in \mathcal{D}(B), \quad u^{1} \in \mathcal{D}(F), \quad f \in C^{1}[0, T ; \mathcal{H}] .
$$

Then the Cauchy problem (2.1) has a unique strong solution on the segment $[0, T]$.

Proof. Since $B$ is semibounded from below, there exist operators $B_{+}, B_{-}$such that

$$
B=B_{+}-B_{-}, \quad B_{+} \geq 0, \quad B_{-}>0, \quad B_{-} \in \mathcal{L}(\mathcal{H}) .
$$

Then problem (2.1) can be rewritten as

$$
\frac{d^{2} u}{d t^{2}}+(F+1 K) \frac{d u}{d t}+B_{+} u=f+B_{-} u=: f_{u}, \quad u(0)=u^{0}, \quad u^{\prime}(0)=u^{1} .
$$

If we replace the operator $B$ by $B_{+}$and the function $f$ by $f_{u}$ in the Cauchy problem (2.1), we can follow the lines of the previous reasoning. Instead of the relations (2.7) - 2.8 we then have

$$
\begin{aligned}
& \frac{d v}{d t}=-{ }_{1} B_{+}^{1 / 2} u, \quad v(0)=0, \\
& \frac{d^{2} v}{d t^{2}}=-{ }_{1} B_{+}^{1 / 2} \frac{d u}{d t}, \quad v^{\prime}(0)=-1 B_{+}^{1 / 2} u^{0},
\end{aligned}
$$

and the analogues of (2.9), (2.10) are

$$
\begin{aligned}
& \frac{d^{2}}{d t^{2}}\left(\begin{array}{l}
u \\
v
\end{array}\right)+\left(\begin{array}{cc}
F+1 K & 1 B_{+}^{1 / 2} \\
1 B_{+}^{1 / 2} & 0
\end{array}\right) \frac{d}{d t}\left(\begin{array}{l}
u \\
v
\end{array}\right)=\left(\begin{array}{c}
f_{u} \\
0
\end{array}\right), \\
& u(0)=u^{0}, \quad u^{\prime}(0)=u^{1}, \quad v(0)=0, \quad v^{\prime}(0)=-1 B_{+}^{1 / 2} u^{0} .
\end{aligned}
$$

Hence, instead of the block operator matrix $\mathcal{A}_{0}$ (see (2.12)) the block operator matrix

$$
\mathcal{A}_{0,+}:=\left(\begin{array}{cc}
F+{ }_{1} K & { }_{1} B_{+}^{1 / 2} \\
{ }_{1} B_{+}^{1 / 2} & 0
\end{array}\right)
$$


arises here, which is defined on the domain

$$
\mathcal{D}\left(\mathcal{A}_{0,+}\right)=\mathcal{D}(F) \oplus \mathcal{D}\left(B_{+}^{1 / 2}\right) .
$$

Using the first condition in (2.46), we see that for every $y=\left(y_{1}, y_{2}\right)^{\mathrm{t}} \in \mathcal{D}\left(\mathcal{A}_{0,+}\right)$,

$$
\operatorname{Re}\left(\mathcal{A}_{0,+} y, y\right)_{\mathcal{H}^{2}}=\left(F y_{1}, y_{1}\right)_{\mathcal{H}} \geq \gamma_{F}\left\|y_{1}\right\|_{\mathcal{H}}^{2} .
$$

Now let $\alpha>0$ be such that

$$
\min \left\{\gamma_{F}+\alpha, \alpha\right\}=: a>0 .
$$

Then the operator

$$
\mathcal{A}_{a,+}:=\mathcal{A}_{0,+}+\alpha \mathcal{I}=\left(\begin{array}{cc}
F_{\alpha}+{ }_{1} K & 1 B_{+}^{1 / 2} \\
1 B_{+}^{1 / 2} & \alpha I
\end{array}\right), \quad F_{\alpha}:=F+\alpha I \geq a I,
$$

is uniformly accretive. Indeed,

$$
\operatorname{Re}\left(\mathcal{A}_{a,+} y, y\right)_{\mathcal{H}^{2}} \geq\left(\gamma_{F}+\alpha\right)\left\|y_{1}\right\|_{\mathcal{H}}^{2}+\alpha\left\|y_{2}\right\|_{\mathcal{H}}^{2} \geq a\|y\|_{\mathcal{H}^{2}}^{2}, y \in \mathcal{D}\left(\mathcal{A}_{a,+}\right) .
$$

Replacing the operators $Q_{a}$ and $Q_{a}^{+}$in (2.20) by

$$
Q_{a,+}:=B_{+}^{1 / 2} F_{\alpha}^{-1 / 2}, \quad Q_{a,+}^{+}:=F_{\alpha}^{-1 / 2} B_{+}^{1 / 2}, \quad \mathcal{D}\left(Q_{a,+}^{+}\right)=\mathcal{D}\left(B_{+}^{1 / 2}\right),
$$

we find that Theorem 2.4 also holds for the operator $\mathcal{A}_{a,+}=\mathcal{A}_{0,+}+\alpha \mathcal{I}$. In particular, $\mathcal{A}_{a,+}$ is closable and its closure

$$
\mathcal{A}_{+}:=\overline{\mathcal{A}_{a,+}}
$$

is maximal uniformly accretive with

$$
\operatorname{Re}\left(\mathcal{A}_{+} y, y\right)_{\mathcal{H}^{2}} \geq a\|y\|_{\mathcal{H}^{2}}^{2}, \quad y \in \mathcal{D}\left(\mathcal{A}_{+}\right),
$$

and we have the analogues of (2.24) $-(2.27)$ :

$$
\begin{aligned}
& \mathcal{A}_{+}=\left(\begin{array}{cc}
I & 0 \\
{ }_{1} Q_{a,+} F_{\alpha}^{-1 / 2} & I
\end{array}\right)\left(\begin{array}{cc}
F_{\alpha} & 0 \\
0 & \alpha I+Q_{a,+} Q_{a,+}^{*}
\end{array}\right)\left(\begin{array}{cc}
I & { }_{1} F_{\alpha}^{-1 / 2} Q_{a,+}^{*} \\
0 & I
\end{array}\right)+\left(\begin{array}{cc}
1 K & 0 \\
0 & 0
\end{array}\right), \\
& \mathcal{A}_{+}=\left(\begin{array}{cc}
F_{\alpha}^{1 / 2} & 0 \\
0 & I
\end{array}\right)\left(\begin{array}{cc}
I+{ }_{1} F_{\alpha}^{-1 / 2} K F_{\alpha}^{-1 / 2} & 1 Q_{a,+}^{*} \\
1 Q_{a,+} & \alpha I
\end{array}\right)\left(\begin{array}{cc}
F_{\alpha}^{1 / 2} & 0 \\
0 & I
\end{array}\right), \\
& \mathcal{D}\left(\mathcal{A}_{+}\right)=\left\{z=\left(z_{1}, z_{2}\right)^{\mathrm{t}} \in \mathcal{H}^{2}: z_{1}+{ }_{1} F_{\alpha}^{-1 / 2} Q_{a,+}^{*} z_{2} \in \mathcal{D}\left(F_{\alpha}\right)\right\},
\end{aligned}
$$

and, for $z \in \mathcal{D}\left(\mathcal{A}_{+}\right)$,

$$
\begin{aligned}
& \mathcal{A}_{+} z=\left(\begin{array}{c}
F_{\alpha}\left(z_{1}+{ }_{1} F_{\alpha}^{-1 / 2} Q_{a,+}^{*} z_{2}\right)+{ }_{1} K z_{1} \\
{ }_{1} Q_{a,+} F_{\alpha}^{1 / 2} z_{1}+\alpha z_{2}
\end{array}\right) \\
& =\left(\begin{array}{c}
F_{\alpha}^{1 / 2}\left(F_{\alpha}^{1 / 2} z_{1}+1 Q_{a,+}^{*} z_{2}\right)+1 K z_{1} \\
1 Q_{a,+} F_{\alpha}^{1 / 2} z_{1}+\alpha z_{2}
\end{array}\right) .
\end{aligned}
$$

In the same way as in Corollary 2.5 we now find that $-\mathcal{A}_{+}$is the generator of a contractive semigroup $\mathcal{U}_{+}(t)$ given by

$$
\mathcal{U}_{+}(t)=\exp \left(-t \mathcal{A}_{+}\right), \quad\left\|\mathcal{U}_{+}(t)\right\| \leq \mathrm{e}^{-a t}, \quad t \geq 0 .
$$

As in (2.16), we introduce a new unknown function $z$ by the relation

$$
y(t)=\left(\frac{d u}{d t}(t), \frac{d v}{d t}(t)\right)^{\mathrm{t}}=\mathrm{e}^{\alpha t} z(t), \quad t \in[0, T]
$$


with $\alpha$ as in (2.51). Then, by (2.48) - (2.49), $z$ is a solution of the Cauchy problem

$$
\frac{d z}{d t}+\mathcal{A}_{a,+} z=\hat{f}_{\alpha, u}, \quad z(0)=z^{0}=y^{0}=\left(u^{1},-1 B_{+}^{1 / 2} u^{0}\right)^{\mathrm{t}},
$$

where

$$
\hat{f}_{\alpha, u}(t):=\mathrm{e}^{-\alpha t} \hat{f}_{0, u}(t):=\mathrm{e}^{-\alpha t}\left(\begin{array}{c}
f_{u}(t) \\
0
\end{array}\right), \quad t \in[0, T] .
$$

As in the proof of Theorem 2.7 we consider the associated Cauchy problem for the closure $\mathcal{A}_{+}$of $\mathcal{A}_{a,+}$,

$$
\frac{d z}{d t}+\mathcal{A}_{+} z=\hat{f}_{\alpha, u}, \quad z(0)=y^{0}=\left(u^{1},-1 B_{+}^{1 / 2} u^{0}\right)^{\mathrm{t}} .
$$

By (2.47), we have $y^{0} \in \mathcal{D}(F) \oplus \mathcal{D}\left(B_{+}^{1 / 2}\right)=\mathcal{D}\left(\mathcal{A}_{a,+}\right) \subset \mathcal{D}\left(\mathcal{A}_{+}\right)$. If $\hat{f}_{\alpha, u} \in$ $C^{1}\left[0, T ; \mathcal{H}^{2}\right]$, then, by Theorem [2.6] the Cauchy problem (2.57) has the unique strong solution

$$
z(t)=\mathcal{U}_{+}(t) z^{0}+\int_{0}^{t} \mathcal{U}_{+}(t-s) \hat{f}_{\alpha, u}(s) d s, \quad t \in[0, T] .
$$

If we represent the function $\hat{f}_{\alpha, u}$ in the form

$$
\begin{aligned}
\hat{f}_{\alpha, u}(t) & =\hat{f}_{\alpha}(t)+\mathrm{e}^{-\alpha t} \widehat{B}_{-}(u(t), v(t))^{\mathrm{t}} \\
& =\hat{f}_{\alpha}(t)+\mathrm{e}^{-\alpha t} \widehat{B}_{-}(u(0), v(0))^{\mathrm{t}}+\mathrm{e}^{-\alpha t} \widehat{B}_{-} \int_{0}^{t}\left(\frac{d u}{d \xi}(\xi), \frac{d v}{d \xi}(\xi)\right)^{\mathrm{t}} d \xi \\
& =\hat{f}_{\alpha}(t)+\mathrm{e}^{-\alpha t} \widehat{B}_{-}\left(u^{0}, 0\right)^{\mathrm{t}}+\mathrm{e}^{-\alpha t} \int_{0}^{t} \widehat{B}_{-} \mathrm{e}^{\alpha \xi} z(\xi) d \xi, \quad t \in[0, T],
\end{aligned}
$$

where

$$
\widehat{B}_{-}:=\operatorname{diag}\left(B_{-}, 0\right) \in \mathcal{L}\left(\mathcal{H}^{2}\right), \quad \hat{f}_{\alpha}(t):=\mathrm{e}^{-\alpha t}(f(t), 0)^{\mathrm{t}},
$$

and substitute this into relation (2.58), we obtain an integral Volterra equation of the second kind for the unknown function $z$ of the form

$$
\begin{aligned}
z(t)= & \mathcal{U}_{+}(t) z^{0}+\int_{0}^{t} \mathcal{U}_{+}(t-s) \hat{f}_{\alpha}(s) d s+\int_{0}^{t} \mathrm{e}^{-\alpha s} \mathcal{U}_{+}(t-s) \widehat{B}_{-}\left(u^{0}, 0\right)^{\mathrm{t}} d s \\
& +\int_{0}^{t} \int_{\xi}^{t} \mathrm{e}^{-\alpha(s-\xi)} \mathcal{U}_{+}(t-s) \widehat{B}_{-} d s z(\xi) d \xi
\end{aligned}
$$

If we denote

$$
\mathcal{V}(t, \xi):=\int_{\xi}^{t} \mathrm{e}^{-\alpha(s-\xi)} \mathcal{U}_{+}(t-s) \widehat{B}_{-} d s
$$

then the kernel function $\mathcal{V}$ is an operator function with values in the space $\mathcal{H}^{2}$ which is continuous in $t$ and $\xi$.

Since $z^{0}=y^{0} \in \mathcal{D}\left(\mathcal{A}_{a,+}\right) \subset \mathcal{D}\left(\mathcal{A}_{+}\right)$and $\hat{f}_{\alpha}+\mathrm{e}^{-\alpha t} \widehat{B}_{-}\left(u^{0}, 0\right)^{\mathrm{t}} \in C^{1}\left[0, T ; \mathcal{H}^{2}\right]$ by assumption (2.47), the function $\varphi$ given by

$$
\varphi(t):=\mathcal{U}_{+}(t) z^{0}+\int_{0}^{t} \mathcal{U}_{+}(t-s) \hat{f}_{\alpha}(s) d s+\int_{0}^{t} \mathcal{U}_{+}(t-s) \mathrm{e}^{-\alpha s} \widehat{B}_{-}\left(u^{0}, 0\right)^{\mathrm{t}} d s
$$

is a strong solution of (2.57) with $\hat{f}_{\alpha, u}$ replaced by $\hat{f}_{\alpha}+\mathrm{e}^{-\alpha t} \widehat{B}_{-}\left(u^{0}, 0\right)^{\mathrm{t}}$ and hence continuously differentiable by Theorem 2.6 . 
Hence, by [KKMRSZ, Theorem 1.8], the Volterra equation (2.59) has a unique solution $z \in C\left[0, T ; \mathcal{H}^{2}\right]$. But $z$ also belongs to $C^{1}\left[0, T ; \mathcal{H}^{2}\right]$ because $\varphi \in C^{1}\left[0, T ; \mathcal{H}^{2}\right]$ and

$$
\begin{aligned}
\frac{d}{d t} \int_{0}^{t} \mathcal{V}(t, \xi) z(\xi) d \xi= & \frac{d}{d t} \int_{0}^{t}\left(\int_{\xi}^{t} \mathcal{U}_{+}(t-s) \widehat{B}_{-} \mathrm{e}^{-\alpha s} d s\right) \mathrm{e}^{\alpha \xi} z(\xi) d \xi \\
= & \frac{d}{d t}\left(\mathrm{e}^{-\alpha t} \int_{0}^{t}\left(\int_{0}^{t-\xi} \mathcal{U}_{+}(\eta) \widehat{B}_{-} \mathrm{e}^{\alpha \eta} d \eta\right) \mathrm{e}^{\alpha \xi} z(\xi) d \xi\right) \\
= & -\alpha \mathrm{e}^{-\alpha t} \int_{0}^{t}\left(\int_{0}^{t-\xi} \mathcal{U}_{+}(\eta) \widehat{B}_{-} \mathrm{e}^{\alpha \eta} d \eta\right) \mathrm{e}^{\alpha \xi} z(\xi) d \xi \\
& +\int_{0}^{t} \mathcal{U}_{+}(t-\xi) \widehat{B}_{-} z(\xi) d \xi
\end{aligned}
$$

which is a continuous function in $t$ on $[0, T]$.

Thus, if assumption (2.47) is satisfied, then $z$ is a solution of equation (2.58) belonging to $C^{1}\left[0, T ; \mathcal{H}^{2}\right]$ and hence a strong solution of the Cauchy problem (2.57).

The last part of the proof follows the lines of Step 4 of the proof of Theorem 2.7 with $\mathcal{A}$ replaced by $\mathcal{A}_{+}$.

2.5. The case of an unbounded Coriolis operator. In this section we consider the Cauchy problem (2.1) for the case when the operator $K$ is unbounded and subordinate to the main operator $F$.

Theorem 2.9. Suppose that there are real constants $\gamma_{F}$ and $\gamma_{B}$ such that

$$
F \geq \gamma_{F} I, \quad B \geq \gamma_{B} I, \quad \mathcal{D}(F) \subset \mathcal{D}(B), \quad K=K^{*}
$$

with

$$
\mathcal{D}(K) \supset \mathcal{D}\left(F_{\alpha}^{1 / 2}\right)
$$

where $\alpha$ is such that $\min \left\{\gamma_{F}+\alpha, \alpha\right\}>0$ as in (2.51). If

$$
u^{0} \in \mathcal{D}(B), \quad u^{1} \in \mathcal{D}(F), \quad f \in C^{1}[0, T ; \mathcal{H}],
$$

then the Cauchy problem (2.1) has a unique strong solution on the segment $[0, T]$.

Proof. By (2.62) the operator $K F_{\alpha}^{-1 / 2}$ is everywhere defined and bounded in $\mathcal{H}$. Therefore we can combine the proofs of Theorem 2.7 and Theorem 2.8 up to formulas (2.53) - (2.56). But these formulas are also valid in the case under consideration here. Indeed, the first representation (2.53) of the operator $\mathcal{A}_{+}$is evident because also here $K F_{\alpha}^{-1 / 2}$ is a bounded operator in $\mathcal{H}$. Therefore the description of the domain of the operator $\mathcal{A}_{+}$in (2.55) remains valid. Moreover, $K z_{1}$ is well defined for any $z=\left(z_{1}, z_{2}\right)^{\mathrm{t}} \in \mathcal{D}\left(\mathcal{A}_{+}\right)$since for these $z$ we have $z_{1} \in \mathcal{D}\left(F_{\alpha}^{1 / 2}\right) \subset \mathcal{D}(K)$ by (2.62). Hence, the second formula in representation (2.53) and formula (2.56) continue to hold as well. Repeating the same arguments as in the proof of Theorem 2.8 after formulas (2.53) - (2.56), we complete the proof of this theorem.

Next we consider the case when the operator $K$ is not subordinate to the operator $F_{\alpha}^{1 / 2}$, but to the operator $F$ itself.

Theorem 2.10. Let conditions (2.61), (2.63) of Theorem 2.9 be fulfilled and let the operator $K=K^{*}$ be unbounded so that

$$
\mathcal{D}(F) \subset \mathcal{D}(K), \quad\left(I+{ }_{1} K F_{\alpha}^{-1}\right)^{-1} \in \mathcal{L}(\mathcal{H})
$$


with $\alpha$ such that $\min \left\{\gamma_{F}+\alpha, \alpha\right\}>0$ as in 2.51) and $F_{\alpha}:=F+\alpha I$. Then the Cauchy problem (2.1) has a unique strong solution on the segment $[0, T]$.

Proof. Here we repeat the proof of Theorem 2.8 up to formula (2.52). First we have to show that the operator $\mathcal{A}_{a,+}$ is closable. For $\mathcal{A}_{a,+}$ we have the representation

$$
\mathcal{A}_{a,+}=\left(\begin{array}{cc}
F_{\alpha}^{1 / 2} & 0 \\
0 & I
\end{array}\right)\left(\begin{array}{cc}
I+1 F_{\alpha}^{-1 / 2} K F_{\alpha}^{-1 / 2} & 1 Q_{a,+}^{+} \\
1 Q_{a,+} & \alpha I
\end{array}\right)\left(\begin{array}{cc}
F_{\alpha}^{1 / 2} & 0 \\
0 & I
\end{array}\right)
$$

on the domain

$$
\mathcal{D}\left(\mathcal{A}_{a,+}\right)=\mathcal{D}(F) \oplus \mathcal{D}\left(B_{+}^{1 / 2}\right)
$$

where

$$
Q_{a,+}:=B_{+}^{1 / 2} F_{\alpha}^{-1 / 2}, \quad Q_{a,+}^{+}:=F_{\alpha}^{-1 / 2} B_{+}^{1 / 2} .
$$

In the sequel we follow the lines of the proof of relation (2.25) in Theorem 2.4 (see the last paragraph of the proof of Theorem 2.4). Also here the outer factors in (2.65) are closed and boundedly invertible, while the operator in the middle

$$
\mathcal{T}_{a,+}:=\left(\begin{array}{cc}
I+{ }_{1} F_{\alpha}^{-1 / 2} K F_{\alpha}^{-1 / 2} & 1 Q_{a,+}^{+} \\
1 Q_{a,+} & \alpha I
\end{array}\right)
$$

is bounded and densely defined. Indeed, using the polar decomposition of $K$, we have

$$
K=J_{K}|K|=|K|^{1 / 2} J_{K}|K|^{1 / 2}
$$

where

$$
J_{K}=J_{K}^{*} \in \mathcal{L}(\mathcal{H}), \quad|K|:=\left(K^{2}\right)^{1 / 2} \geq 0, \quad \mathcal{D}(K)=\mathcal{D}(|K|) .
$$

Thus the operator $F_{\alpha}^{-1 / 2} K F_{\alpha}^{-1 / 2}$, which is densely defined, can be written as

$$
F_{\alpha}^{-1 / 2} K F_{\alpha}^{-1 / 2}=\left(F_{\alpha}^{-1 / 2}|K|^{1 / 2}\right) J_{K}\left(|K|^{1 / 2} F_{\alpha}^{-1 / 2}\right)=V_{\alpha}^{+} J_{K} V_{\alpha}
$$

with

$$
V_{\alpha}:=|K|^{1 / 2} F_{\alpha}^{-1 / 2}, \quad V_{\alpha}^{+}:=F_{\alpha}^{-1 / 2}|K|^{1 / 2} .
$$

Using the first condition in (2.64) and Lemma 2.3 (with $|K|$ instead of $B$ ), we conclude

$$
V_{\alpha} \in \mathcal{L}(\mathcal{H}), \quad \mathcal{D}\left(V_{\alpha}^{+}\right)=\mathcal{D}\left(|K|^{1 / 2}\right) \supset \mathcal{D}(|K|)=\mathcal{D}(K), \quad V_{\alpha}^{+}=V_{\alpha}^{*} \mid \mathcal{D}\left(|K|^{1 / 2}\right) .
$$

Therefore the operator $F_{\alpha}^{-1 / 2} K F_{\alpha}^{-1 / 2}$ is bounded and hence closable with closure

$$
\overline{F_{\alpha}^{-1 / 2} K F_{\alpha}^{-1 / 2}}=V_{\alpha}^{*} J_{K} V_{\alpha} \in \mathcal{L}(\mathcal{H}) .
$$

Thus $\mathcal{T}_{a,+}$ is bounded and densely defined and its closure is obtained by taking the closure of $F_{\alpha}^{-1 / 2} K F_{\alpha}^{-1 / 2}$ and of $Q_{a,+}$. Moreover, for the closure of $\mathcal{T}_{a,+}$ we have

$$
\operatorname{Re}\left(\overline{\mathcal{T}_{a,+}} y, y\right)_{\mathcal{H}^{2}}=\left(y_{1}, y_{1}\right)_{\mathcal{H}}+\alpha\left(y_{2}, y_{2}\right)_{\mathcal{H}} \geq \min \{1, \alpha\}\|y\|_{\mathcal{H}^{2}}^{2}
$$

for $y=\left(y_{1}, y_{2}\right)^{\mathrm{t}} \in \mathcal{H}^{2}$. In the same way as in the end of the proof of Theorem 2.4 we now find that the operator $\mathcal{A}_{a,+}$ is closable and its closure $\mathcal{A}_{+}$has the form

$$
\mathcal{A}_{+}=\left(\begin{array}{cc}
F_{\alpha}^{1 / 2} & 0 \\
0 & I
\end{array}\right)\left(\begin{array}{cc}
I+{ }_{1} V_{\alpha}^{*} J_{K} V_{\alpha} & 1 Q_{a,+}^{*} \\
1 Q_{a,+} & \alpha I
\end{array}\right)\left(\begin{array}{cc}
F_{\alpha}^{1 / 2} & 0 \\
0 & I
\end{array}\right)
$$

with

$\mathcal{D}\left(\mathcal{A}_{+}\right)=\left\{z=\left(z_{1}, z_{2}\right)^{\mathrm{t}} \in \mathcal{H}^{2}: z_{1}+{ }_{1} F_{\alpha}^{-1 / 2} V_{\alpha}^{*} J_{K} V_{\alpha} F_{\alpha}^{1 / 2} z_{1}+{ }_{1} F_{\alpha}^{-1 / 2} Q_{a,+}^{*} z_{2} \in \mathcal{D}\left(F_{\alpha}\right)\right\}$ 
and

$$
\mathcal{A}_{+} z=\left(\begin{array}{c}
F_{\alpha}\left(z_{1}+{ }_{1} F_{\alpha}^{-1 / 2} V_{\alpha}^{*} J_{K} V_{\alpha} F_{\alpha}^{1 / 2} z_{1}+{ }_{1} F_{\alpha}^{-1 / 2} Q_{a,+}^{*} z_{2}\right) \\
{ }_{1} Q_{a,+} F_{\alpha}^{1 / 2} z_{1}+\alpha z_{2}
\end{array}\right) .
$$

Using the first equality in (2.68), we see that

$\operatorname{Re}\left(\mathcal{A}_{+} z, z\right)_{\mathcal{H}^{2}}=\left(F_{\alpha} z_{1}, z_{1}\right)_{\mathcal{H}}+\alpha\left(z_{2}, z_{2}\right)_{\mathcal{H}} \geq a\|z\|_{\mathcal{H}^{2}}^{2}, \quad z=\left(z_{1}, z_{2}\right)^{\mathrm{t}} \in \mathcal{D}\left(\mathcal{A}_{+}\right)$.

Therefore and because $\overline{\mathcal{T}_{a,+}}$ is maximal uniformly accretive, the same is true for $\mathcal{A}_{+}$.

Now we can repeat the same arguments as in the proof of Theorem 2.8 from the statement of the Cauchy problem (2.57) up to the assertion after formula (2.60) that the function $z$ belongs to $C^{1}\left[0, T ; \mathcal{H}^{2}\right]$ and is a strong solution of (2.57).

Here this means that $z=\left(z_{1}, z_{2}\right)^{\mathrm{t}}$ satisfies the system of equations

$$
\begin{aligned}
& \frac{d z_{1}}{d t}(t)+F_{\alpha}\left(z_{1}(t)+1 F_{\alpha}^{-1 / 2} V_{\alpha}^{*} J_{K} V_{\alpha} F_{\alpha}^{1 / 2} z_{1}(t)+1 F_{\alpha}^{-1 / 2} Q_{a,+}^{*} z_{2}(t)\right)=\mathrm{e}^{-\alpha t} f_{u}(t), \\
& \frac{d z_{2}}{d t}(t)+\alpha z_{2}(t)+{ }_{1} Q_{a,+} F_{\alpha}^{1 / 2} z_{1}(t)=0, \quad z_{1}(0)=u^{1}, \quad z_{2}(0)=-1 B_{+}^{1 / 2} u^{0}
\end{aligned}
$$

for any $t \in[0, T]$ where

$$
z_{i}(t)=\mathrm{e}^{-\alpha t} y_{i}(t), \quad i=1,2, \quad y_{1}=u^{\prime}, \quad y_{2}=v^{\prime}={ }_{-1} B_{+}^{1 / 2} u, \quad f_{u}=f+B_{-} u
$$

From the second equation in (2.69) we conclude

$$
z_{2}(t)=-1 \mathrm{e}^{-\alpha t} B_{+}^{1 / 2} u^{0}-1 \int_{0}^{t} \mathrm{e}^{-\alpha(t-s)} Q_{a,+} F_{\alpha}^{1 / 2} z_{1}(s) d s .
$$

Substituting this into the first equation in (2.69), we obtain

$$
\begin{aligned}
& \frac{d z_{1}}{d t}(t)+F_{\alpha}\left(z_{1}(t)+{ }_{1} F_{\alpha}^{-1 / 2} V_{\alpha}^{*} J_{K} V_{\alpha} F_{\alpha}^{1 / 2} z_{1}(t)+F_{\alpha}^{-1 / 2} Q_{a,+}^{*} B_{+}^{1 / 2} u^{0} \mathrm{e}^{-\alpha t}\right. \\
& \left.+F_{\alpha}^{-1 / 2} Q_{a,+}^{*} \int_{0}^{t} \mathrm{e}^{-\alpha(t-s)} Q_{a,+} F_{\alpha}^{1 / 2} z_{1}(s) d s\right)=\mathrm{e}^{-\alpha t} f_{u}(t), \quad z_{1}(0)=u^{1} .
\end{aligned}
$$

Here the function $\varphi$ given by

$$
\begin{aligned}
\varphi(t):= & z_{1}(t)+{ }_{1} F_{\alpha}^{-1 / 2} V_{\alpha}^{*} J_{K} V_{\alpha} F_{\alpha}^{1 / 2} z_{1}(t)+F_{\alpha}^{-1 / 2} Q_{a,+}^{*} B_{+}^{1 / 2} u^{0} \mathrm{e}^{-\alpha t} \\
& +F_{\alpha}^{-1 / 2} Q_{a,+}^{*} \int_{0}^{t} \mathrm{e}^{-\alpha(t-s)} Q_{a,+} F_{\alpha}^{1 / 2} z_{1}(s) d s, \quad t \in[0, T],
\end{aligned}
$$

is continuous with values in $\mathcal{D}\left(F_{\alpha}\right)=\mathcal{D}(F)$ since $z \in \mathcal{D}\left(\mathcal{A}_{+}\right)$. But the same is true for the function $\varphi_{0}$ given by

$$
\varphi_{0}(t):=F_{\alpha}^{-1 / 2} Q_{a,+}^{*} B_{+}^{1 / 2} u^{0} \mathrm{e}^{-\alpha t}, \quad t \in[0, T]
$$

if $u^{0} \in \mathcal{D}\left(B_{+}\right)=\mathcal{D}(B)$ (see the end of Step 2 in the proof of Theorem 2.7 and formulas (2.41), (2.42) $)$. Hence, relation (2.72) can be rewritten in the form

$$
\begin{aligned}
& z_{1}(t)+{ }_{1} F_{\alpha}^{-1 / 2} V_{\alpha}^{*} J_{K} V_{\alpha} F_{\alpha}^{1 / 2} z_{1}(t)+F_{\alpha}^{-1 / 2} Q_{a,+}^{*} \int_{0}^{t} \mathrm{e}^{-\alpha(t-s)} Q_{a,+} F_{\alpha}^{1 / 2} z_{1}(s) d s \\
& =\varphi(t)-\varphi_{0}(t)=: \varphi_{1}(t), \quad t \in[0, T]
\end{aligned}
$$

where $\varphi_{1} \in C\left[0, T ; \mathcal{D}\left(F_{\alpha}\right)\right]$,

$$
\varphi_{1}=F_{\alpha}^{-1} \eta \quad \text { for some } \eta \in C[0, T ; \mathcal{H}] .
$$


Now consider the equation

$$
\begin{aligned}
& \psi(t)+{ }_{1} F_{\alpha}^{1 / 2} V_{\alpha}^{*} J_{K} V_{\alpha} F_{\alpha}^{-1 / 2} \psi(t) \\
& +\int_{0}^{t} \mathrm{e}^{-\alpha(t-s)} F_{\alpha}^{1 / 2} Q_{a,+}^{*} Q_{a,+} F_{\alpha}^{-1 / 2} \psi(s) d s=\eta(t), \quad t \in[0, T] .
\end{aligned}
$$

As in Step 3 and formula (2.44) in the proof of Theorem 2.7, we see that

$$
F_{\alpha}^{-1 / 2} Q_{a,+}^{*} Q_{a,+} F_{\alpha}^{-1 / 2}=F_{\alpha}^{-1 / 2} Q_{a,+}^{+} B_{+}^{1 / 2} F_{\alpha}^{-1}=F_{\alpha}^{-1} B_{+} F_{\alpha}^{-1} .
$$

Further, since $\mathcal{D}(F) \subset \mathcal{D}(K)$ by assumption (2.64), we have

$$
|K|^{1 / 2} F_{\alpha}^{-1} \psi \in \mathcal{D}\left(|K|^{1 / 2}\right)=\mathcal{D}\left(J_{K}|K|^{1 / 2}\right)=\mathcal{D}\left(|K|^{1 / 2} J_{K}\right)
$$

and hence

$$
\begin{aligned}
F_{\alpha}^{-1 / 2} V_{\alpha}^{*} J_{K} V_{\alpha} F_{\alpha}^{-1 / 2} \psi & =F_{\alpha}^{-1 / 2} V_{\alpha}^{*} J_{K}|K|^{1 / 2} F_{\alpha}^{-1} \psi \\
& =F_{\alpha}^{-1 / 2} V_{\alpha}^{+} J_{K}|K|^{1 / 2} F_{\alpha}^{-1} \psi \\
& =F_{\alpha}^{-1}|K|^{1 / 2} J_{K}|K|^{1 / 2} F_{\alpha}^{-1} \psi \\
& =F_{\alpha}^{-1} K F_{\alpha}^{-1} \psi .
\end{aligned}
$$

Thus relation (2.73) is equivalent to

$$
\psi(t)+{ }_{1} K F_{\alpha}^{-1} \psi(t)+\int_{0}^{t} \mathrm{e}^{-\alpha(t-s)} B_{+} F_{\alpha}^{-1} \psi(s) d s=\eta(t), \quad t \in[0, T] .
$$

Here, by (2.64) and (2.4), the operators $K F_{\alpha}^{-1}$ and $B_{+} F_{\alpha}^{-1}$ are bounded and the operator $I+{ }_{1} K F_{\alpha}^{-1}$ has a bounded inverse. Therefore relation (2.76) is equivalent to

$$
\psi(t)+\int_{0}^{t} \mathrm{e}^{-\alpha(t-s)}\left(I+{ }_{1} K F_{\alpha}^{-1}\right)^{-1} B_{+} F_{\alpha}^{-1} \psi(s) d s=\left(I+1 K F_{\alpha}^{-1}\right)^{-1} \eta(t)
$$

for $t \in[0, T]$, i.e., to a Volterra integral equation of the second kind with operator valued kernel function

$$
\mathrm{e}^{-\alpha(t-s)}\left(I+{ }_{1} K F_{\alpha}^{-1}\right)^{-1} B_{+} F_{\alpha}^{-1},
$$

which is continuous in $t$ and $s$, and with continuous right-hand side $\left(I+{ }_{1} K F_{\alpha}^{-1}\right)^{-1} \eta$. Therefore (2.77) (and thus also (2.73)) has a unique continuous solution $\psi$. Then (2.72) has the unique solution $z_{1}:=F_{\alpha}^{-1} \psi \in C\left[0, T ; \mathcal{D}\left(F_{\alpha}\right)\right]$, and therefore we can open the brackets in equation (2.71). From (2.74), (2.75) we then obtain

$$
\begin{aligned}
& F_{\alpha}^{1 / 2} V_{\alpha}^{*} J_{K} V_{\alpha} F_{\alpha}^{1 / 2} z_{1}=K z_{1}, \quad F_{\alpha}^{1 / 2} Q_{a,+}^{*} B_{+}^{1 / 2} u^{0}=B_{+} u^{0} \quad \text { if } u^{0} \in \mathcal{D}\left(B_{+}\right)=\mathcal{D}(B), \\
& \int_{0}^{t} \mathrm{e}^{-\alpha(t-s)} F_{\alpha}^{1 / 2} Q_{a,+}^{*} Q_{a,+} F_{\alpha}^{1 / 2} z_{1}(s) d s=\mathrm{e}^{-\alpha t} \int_{0}^{t} \mathrm{e}^{\alpha s} B_{+} z_{1}(s) d s, \quad t \in[0, T] .
\end{aligned}
$$

Using these relations and the equations $z_{1}(t)=\mathrm{e}^{-\alpha t} d u(t) / d t, t \in[0, T], f_{u}=$ $f+B_{-} u$ (see (2.70) $)$ in the differential equation (2.71), we see that the function $u$ is a solution of

$$
\frac{d^{2} u}{d t^{2}}+\left(F_{\alpha}-\alpha+1 K\right) \frac{d u}{d t}+B_{+} u=f+B_{-} u,
$$

that is, of the Cauchy problem (2.1), and each term in the above equation is continuous in $t$ with values in the space $\mathcal{H}$.

Remark 2.11. It is evident from the proof of Theorem 2.10 that its assertion is valid whenever equation (2.76) has a unique solution $\psi \in C[0, T ; \mathcal{H}]$. 
Remark 2.12. The operator $I+{ }_{1} K F_{\alpha}^{-1}$ is invertible in $\mathcal{H}$ (with possibly unbounded inverse). Indeed, if $x \in \mathcal{H}$ is such that $\left(I+1 K F_{\alpha}^{-1}\right) x=0$, then

$$
\left(F_{\alpha}^{-1} x, x\right)_{\mathcal{H}}+1\left(F_{\alpha}^{-1} K F_{\alpha}^{-1} x, x\right)_{\mathcal{H}}=0
$$

and hence $x=0$ since $F_{\alpha}^{-1}>0$ and $K=K^{*}$.

Corollary 2.13. The operator $I+{ }_{1} K F_{\alpha}^{-1}$ has a bounded inverse in any of the following cases:

i) The estimate $\left\|K F_{\alpha}^{-1}\right\|<1$ holds.

ii) The operator $K F_{\alpha}^{-1}$ is compact.

iii) The operators $K$ and $F$ commute.

Proof. If i) holds, the assertion is evident. If ii) holds, the inverse $\left(I+{ }_{1} K F_{\alpha}^{-1}\right)^{-1}$ has the form $I+S$ where $S$ is a compact operator by Remark 2.12 and Fredholm theory (see, e.g., GGK, Theorem XI.5.2]). If iii) holds, then, by (2.67), the bounded selfadjoint operator $K F_{\alpha}^{-1}$ can be rewritten in the form $K F_{\alpha}^{-1}=V_{\alpha} J_{K} V_{\alpha}$ (note that $\left.V_{\alpha}=|K|^{1 / 2} F_{\alpha}^{1 / 2}=V_{\alpha}^{*}\right)$. Hence

$$
\operatorname{Re}\left(\left(I \pm{ }_{1} K F_{\alpha}^{-1}\right) x, x\right)_{\mathcal{H}}=\operatorname{Re}\left(\left(I \pm{ }_{1} V_{\alpha} J_{K} V_{\alpha}\right) x, x\right)_{\mathcal{H}}=\|x\|_{\mathcal{H}}^{2}, \quad x \in \mathcal{H},
$$

which shows that $I+{ }_{1} K F_{\alpha}^{-1}$ is accretive with deficiency 0 and thus has a bounded inverse with $\left\|\left(I+{ }_{1} K F_{\alpha}^{-1}\right)^{-1}\right\| \leq 1$.

Remark 2.14. If the condition $u^{0} \in \mathcal{D}(B)$ is strengthened to $u^{0} \in \mathcal{D}(F) \subset \mathcal{D}(B)$, then a strong solution $u$ of problem (2.1) belongs to $C[0, T ; \mathcal{D}(F)]$. Indeed, by Definition [2.1 for such a solution we have $F d u / d t \in C[0, T ; \mathcal{H}]$ and therefore

$$
\int_{0}^{t} F \frac{d u}{d \xi} d \xi=F \int_{0}^{t} \frac{d u}{d \xi} d \xi=F(u(t)-u(0))
$$

belongs to $C[0, T ; \mathcal{H}]$. If $u(0)=u^{0} \in \mathcal{D}(F)$, then also $F u \in C[0, T ; \mathcal{H}]$.

Remark 2.15. Suppose $K=0$. If

$$
u^{0} \in \mathcal{D}(F), \quad u^{1} \in \mathcal{D}(F), \quad f \in C^{1}[0, T ; \mathcal{H}],
$$

then the Cauchy problem (2.1) is stable with respect to any substitution

$$
u(t)=\mathrm{e}^{a t} v(t), \quad a>0 .
$$

This means, after this substitution a strong solution $u$ is transformed into a strong solution $v$ of the transformed Cauchy problem corresponding to equation (2.1). If we only suppose that $u^{0} \in \mathcal{D}(B)$, then a strong solution $u$ may be transformed into a solution $v$ which may not be a strong solution.

\section{HydRodynamicAl APPLICATIONS:}

\section{Small motions of a VISCOUS FLUID IN AN OPEN VESSEL}

As an application of the general approach presented in Section 2, we consider the famous problem of S. G. Krein on small motions of a viscous fluid in an arbitrary open vessel (see [K1], [K2], [KL], [Ko], [KKN]). 
3.1. Statement of the problem. Assume that a rigid immovable vessel is partially filled with a heavy viscous incompressible homogeneous fluid with density $\rho>0$ and kinematic viscosity $\nu>0$. Let $\Omega \subset \mathbb{R}^{3}$ be the region filled by the fluid in equilibrium state, let $S$ be the rigid wall of the vessel adherent to the fluid, and let $\Gamma$ be the free surface of the fluid. We choose the origin $O$ of our Cartesian coordinate system with axes $x_{1}, x_{2}, x_{3}$ on $\Gamma$ so that the acceleration $\mathbf{g}$ due to the gravitational field perpendicular to $\Gamma$ is given by $\mathbf{g}=-g \mathbf{e}_{3}, g>0$, and the equation of the surface $\Gamma$ has the form $x_{3}=0$.

We denote by $P_{0}=P_{0}(x), x=\left(x_{1}, x_{2}, x_{3}\right) \in \Omega$, the pressure in the fluid in equilibrium state. Then $-\rho^{-1} \nabla P_{0}=g \mathbf{e}_{3}$, and so, if $p_{a}$ is the constant external pressure, then

$$
P_{0}=P_{0}\left(x_{3}\right)=-\rho g x_{3}+p_{a} .
$$

We consider small motions of the fluid close to the equilibrium state. We assume that the difference $p=p(t, x)$ between the full pressure $P(t, x)$ and the static pressure $P_{0}\left(x_{3}\right)$ is an infinitesimal function. By $\mathbf{w}=\mathbf{w}(t, x)$ we denote the field of small displacements in the fluid and by $\mathbf{f}=\mathbf{f}(t, x)$ the small field of external mass forces which acts together with the gravitational field in the process of small motions. In the sequel, we suppose that the functions $\mathbf{w}, p$, and $\mathbf{f}$ are infinitesimal of first order.

The initial boundary value problem for the linearized Navier-Stokes equations has the form (see, for instance, [KKN, p. 276]):

$$
\begin{aligned}
& \frac{\partial^{2} \mathbf{w}}{\partial t^{2}}=-\frac{1}{\rho} \nabla p+\nu \Delta \frac{\partial \mathbf{w}}{\partial t}+\mathbf{f}, \quad \operatorname{div} \mathbf{w}=0 \quad \text { in } \Omega \\
& \mathbf{w}=\mathbf{0} \quad \text { on } S, \quad \nu\left(\frac{\partial}{\partial x_{i}} \frac{\partial w_{3}}{\partial t}+\frac{\partial}{\partial x_{3}} \frac{\partial w_{i}}{\partial t}\right)=0 \quad \text { on } \Gamma, \quad i=1,2 \\
& -p+2 \rho \nu \frac{\partial}{\partial x_{3}} \frac{\partial w_{3}}{\partial t}=-\rho g \gamma_{n} \mathbf{w} \quad \text { on } \Gamma, \quad \gamma_{n} \mathbf{w}:=\mathbf{w} \cdot \mathbf{n}=\mathbf{w} \cdot \mathbf{e}_{3} \\
& \mathbf{w}(0, x)=\mathbf{w}^{0}(x), \quad \frac{\partial}{\partial t} \mathbf{w}(0, x)=\mathbf{w}^{1}(x)
\end{aligned}
$$

Here $\mathbf{n}=\mathbf{e}_{3}$ is the unit vector of the external normal to the boundary $\Gamma \subset \partial \Omega$. We suppose that the displacement field $\mathbf{w}$ is solenoídal in $\Omega$ (see the second equation in (3.1) ) and an adhesion condition is fulfilled on $S$ (see the first condition in (3.2)). The second condition in (3.2) and condition (3.3) are dynamical conditions which mean that the tangent and normal stresses on the moving free surface of the fluid equal zero, i.e., they equal the corresponding stresses in the gas located above the fluid. Conditions (3.4) are the initial data for the displacement field and the velocity field of the fluid.

3.2. General spaces and their decompositions. In order to formulate problem (3.1) - (3.4) as a Cauchy problem of the form (2.1), we suppose that the boundary $\partial \Omega$ of the region $\Omega \subset \mathbb{R}^{3}$ is a piecewise smooth surface with nonzero inner and outer angles. In the following we introduce a suitable Hilbert space setting and we consider some auxiliary boundary value problems.

We assume that the unknown fields $\mathbf{w}$ and $\nabla p$ are functions of $t$ with values $\mathbf{w}(\cdot, t)$ and $\nabla p(\cdot, t)$ in the Hilbert space $\mathbf{L}_{2}(\Omega)$ of vector-functions with scalar 
product

$$
(\mathbf{u}, \mathbf{v}):=\int_{\Omega} \mathbf{u}(x) \cdot \overline{\mathbf{v}(x)} d \Omega, \quad \mathbf{u}, \mathbf{v} \in \mathbf{L}_{2}(\Omega) .
$$

We will also need the Hilbert space $L_{2}(\Gamma)$ (of scalar functions) with corresponding scalar product

$$
(\varphi, \psi)_{0}:=\int_{\Gamma} \varphi(x) \overline{\psi(x)} d \Gamma, \quad \varphi, \psi \in L_{2}(\Gamma) .
$$

For a smooth solenoïdal field w we have, by Gauß' Theorem,

$$
0=\int_{\Omega} \operatorname{div} \mathbf{w} d \Omega=\int_{S} \mathbf{w} \cdot \mathbf{n} d S+\int_{\Gamma} \gamma_{n} \mathbf{w} d \Gamma .
$$

Therefore any solution $\mathbf{w}$ of problem (3.1) - (3.4) satisfies

$$
\int_{\Gamma} \gamma_{n} \mathbf{w} d \Gamma=0
$$

i.e., if $\gamma_{n} \mathbf{w} \in L_{2}(\Gamma)$, then $\gamma_{n} \mathbf{w} \in L_{2}(\Gamma) \ominus\left\{1_{\Gamma}\right\}$ where $1_{\Gamma}$ is the unit function on $\Gamma$.

In the sequel we will use the orthogonal decomposition

$$
\mathbf{L}_{2}(\Omega)=\mathbf{J}_{0}(\Omega) \oplus \mathbf{G}_{h, S}(\Omega) \oplus \mathbf{G}_{0, \Gamma}(\Omega),
$$

which is naturally related to the problem of small oscillations of a viscous fluid in an open vessel and which is obtained by means of the so-called Weyl decomposition (see, for instance, [La, Section 1.2], [T, Theorem 1.5, (1.40)], [KKN, p. 106]). Here

$$
\begin{aligned}
\mathbf{J}_{0}(\Omega) & :=\left\{\mathbf{u} \in \mathbf{L}_{2}(\Omega): \operatorname{div} \mathbf{u}=0 \text { in } \Omega, u_{n}:=\mathbf{u} \cdot \mathbf{n}=0 \text { on } \partial \Omega\right\} \\
\mathbf{G}_{h, S}(\Omega) & :=\left\{\mathbf{v} \in \mathbf{L}_{2}(\Omega): \mathbf{v}=\nabla p, \Delta p=0 \text { in } \Omega, \frac{\partial p}{\partial n}=0 \text { on } S, \int_{\Gamma} p d \Gamma=0\right\} \\
\mathbf{G}_{0, \Gamma}(\Omega) & :=\left\{\mathbf{w} \in \mathbf{L}_{2}(\Omega): \mathbf{w}=\nabla \varphi \text { in } \Omega, \varphi=0 \text { on } \Gamma\right\}
\end{aligned}
$$

$\operatorname{div} \mathbf{u}, u_{n}$, and $\partial p / \partial n$ being distributions of finite order (see, e.g., $\mathrm{KKN}$, pp. 100102] or [AHKM Section 2.5]).

From (3.1) and the first boundary condition in (3.2) it follows that for a solution $\{\mathbf{w} ; \nabla p\}$ of (3.1) - 3.4 we have

$$
\begin{aligned}
\mathbf{w} \in \mathbf{J}_{0}(\Omega) \oplus \mathbf{G}_{h, S}(\Omega)=: \mathbf{J}_{0, S}(\Omega) & =\left\{\mathbf{u} \in \mathbf{L}_{2}(\Omega): \operatorname{div} \mathbf{u}=0 \text { in } \Omega, u_{n}=0 \text { on } S\right\}, \\
\nabla p & \in \mathbf{G}_{h, S}(\Omega) \oplus \mathbf{G}_{0, \Gamma}(\Omega)=: \mathbf{G}(\Omega) \\
& =\left\{\mathbf{v} \in \mathbf{L}_{2}(\Omega): \mathbf{v}=\nabla p \text { in } \Omega, \int_{\Gamma} p d \Gamma=0\right\} .
\end{aligned}
$$

3.3. Orthogonal projection approach. We introduce the complementary orthoprojections $P_{0, S}$ and $P_{0, \Gamma}$ from the space $\mathbf{L}_{2}(\Omega)=\mathbf{J}_{0, S}(\Omega) \oplus \mathbf{G}_{0, \Gamma}(\Omega)$ onto the subspaces $\mathbf{J}_{0, S}(\Omega)$ and $\mathbf{G}_{0, \Gamma}(\Omega)$, respectively, (so that $P_{0, S}+P_{0, \Gamma}=I$ ), and we suppose that the problem (3.1) - (3.4) has a solution $\{\mathbf{w} ; \nabla p\}$ for which all terms in the first equation of (3.1) are continuous functions in $t$ with values in $\mathbf{L}_{2}(\Omega)$. Then, by (3.10) and (3.11),

$$
\begin{aligned}
& P_{0, S} \mathbf{w}=\mathbf{w}, P_{0, \Gamma} \mathbf{w}=0, \\
& P_{0, S} \nabla p=: \nabla \tilde{p} \in \mathbf{G}_{h, S}(\Omega), P_{0, \Gamma} \nabla p=: \nabla \varphi \in \mathbf{G}_{0, \Gamma}(\Omega) .
\end{aligned}
$$


Applying the orthoprojections $P_{0, \Gamma}$ and $P_{0, S}$ to both sides of the first equation in (3.1) we obtain, by (3.12),

$$
\begin{aligned}
0 & =-\frac{1}{\rho} \nabla \varphi+\nu P_{0, \Gamma} \Delta \frac{\partial \mathbf{w}}{\partial t}+P_{0, \Gamma} \mathbf{f}, \\
\frac{\partial^{2} \mathbf{w}}{\partial t^{2}} & =-\frac{1}{\rho} \nabla \tilde{p}+\nu P_{0, S} \Delta \frac{\partial \mathbf{w}}{\partial t}+P_{0, S} \mathbf{f} .
\end{aligned}
$$

Relation (3.13) shows that if the displacement field $\mathbf{w}$ is known, then the field $\nabla \varphi$ can be calculated immediately and hence also $\varphi$, using the fact that $\varphi=0$ on $\Gamma$ (see (3.9)). Therefore it is sufficient to investigate the following initial boundary value problem for the unknown functions $\mathbf{w}$ and $\tilde{p}$ :

$$
\begin{aligned}
& \frac{\partial^{2} \mathbf{w}}{\partial t^{2}}=-\frac{1}{\rho} \nabla \tilde{p}+\nu P_{0, S} \Delta \frac{\partial \mathbf{w}}{\partial t}+P_{0, S} \mathbf{f}, \quad \operatorname{div} \mathbf{w}=0 \quad \text { in } \Omega \\
& \mathbf{w}=0 \quad \text { on } S, \quad \nu\left(\frac{\partial}{\partial x_{i}} \frac{\partial w_{3}}{\partial t}+\frac{\partial}{\partial x_{3}} \frac{\partial w_{i}}{\partial t}\right)=0 \quad \text { on } \Gamma, \quad i=1,2 \\
& -\tilde{p}+2 \rho \nu \frac{\partial}{\partial x_{3}} \frac{\partial w_{3}}{\partial t}=-\rho g \gamma_{n} \mathbf{w} \quad \text { on } \Gamma \\
& \mathbf{w}(0, x)=\mathbf{w}^{0}(x), \quad \frac{\partial}{\partial t} \mathbf{w}(0, x)=\mathbf{w}^{1}(x)
\end{aligned}
$$

Here every term in the first equation is assumed to be a function of the variable $t$ with values in the subspace $\mathbf{J}_{0, S}(\Omega)$.

3.4. Auxiliary boundary value problems. For the first auxiliary problem, we introduce the Hilbert space $H_{\Gamma}^{1 / 2}:=H^{1 / 2}(\Gamma) \cap H$, where $H:=L_{2}(\Gamma) \ominus\left\{1_{\Gamma}\right\}$, and the space $H_{\Gamma}^{1}(\Omega)$ which consists of scalar functions $p \in H^{1}(\Omega)$ such that $\int_{\Gamma} p d \Gamma=0$ equipped with the norm

$$
\|p\|_{H_{\Gamma}^{1}(\Omega)}^{2}:=\int_{\Omega}|\nabla p|^{2} d \Omega
$$

Evidently, $H_{\Gamma}^{1}(\Omega)$ is a subspace of codimension 1 of the Sobolev space $H^{1}(\Omega)$, and the Dirichlet norm (3.19) is equivalent to the standard norm of $H^{1}(\Omega)$.

The first auxiliary problem: Zaremba problem for the Laplace equation. Solve the following boundary value problem for the unknown function $p_{1} \in$ $H_{\Gamma}^{1}(\Omega)$ :

$$
\Delta p_{1}=0 \quad \text { in } \Omega, \quad \frac{\partial p_{1}}{\partial n}=0 \quad \text { on } S, \quad p_{1}=\psi \quad \text { on } \Gamma, \quad \int_{\Gamma} \psi d \Gamma=0 .
$$

Lemma 3.1. For every $\psi \in H_{\Gamma}^{1 / 2}$, problem (3.20) has a unique (generalized) solution $p_{1} \in H_{\Gamma}^{1}(\Omega), \nabla p_{1}=: G \psi \in \mathbf{G}_{h, S}(\Omega)$ and

$$
\|G \psi\|=\left\|\nabla p_{1}\right\|=\left\|p_{1}\right\|_{H_{\Gamma}^{1}(\Omega)} \leq c\|\psi\|_{H_{\Gamma}^{1 / 2}} .
$$

Proof. See [KKN, p. 45] and also [AHKM, Lemma 2.4].

Remark 3.2. It follows from the Trace Theorem (see, for instance, $[\mathrm{G}]$ ) that $\|\psi\|_{H_{\Gamma}^{1 / 2}}$ $\leq \hat{c}\|G \psi\|$. Therefore the norm $\|\cdot\|_{H_{\Gamma}^{1 / 2}}$ in the space $H_{\Gamma}^{1 / 2}$ is equivalent to the norm $\|G \cdot\|$. If we equip $H_{\Gamma}^{1 / 2}$ with the latter norm, then, obviously, the operator $G: H_{\Gamma}^{1 / 2} \rightarrow \mathbf{G}_{h, S}(\Omega)$ is an isometry. 
For the second auxiliary problem, we introduce the subspace

$$
\mathbf{J}_{0, S}^{1}(\Omega):=\left\{\mathbf{u} \in \mathbf{H}^{1}(\Omega): \operatorname{div} \mathbf{u}=0 \text { in } \Omega, \mathbf{u}=0 \text { on } S\right\}
$$

of the space $\mathbf{H}^{1}(\Omega)$ of vector fields and equip $\mathbf{J}_{0, S}^{1}(\Omega)$ with the quadratic form

$$
E(\mathbf{u}, \mathbf{u}):=\frac{1}{2} \int_{\Omega} \sum_{i, j=1}^{3}\left|\frac{\partial u_{i}}{\partial x_{j}}+\frac{\partial u_{j}}{\partial x_{i}}\right|^{2} d \Omega, \quad \mathbf{u} \in \mathbf{J}_{0, S}^{1}(\Omega) .
$$

If $\mathbf{u}$ is a velocity field of a viscous fluid, then the form $\rho \nu E(\mathbf{u}, \mathbf{u})$ is the dissipation velocity of the energy in the region $\Omega$. We mention that on the subspace $\mathbf{J}_{0, S}^{1}(\Omega)$ the norm $E(\mathbf{u}, \mathbf{u})^{1 / 2}$ induced by $E$ is equivalent to the standard norm of $\mathbf{H}^{1}(\Omega)$, which can be seen using [KKN], Section 2.2, (2.16)] and Korn's inequality (see [Gob]).

We will also need Green's formula which is directly related to the right-hand side of the first equation in (3.15). Namely, if $\mathbf{u} \in \mathbf{J}_{0, S}^{1}(\Omega) \cap \mathbf{H}^{2}(\Omega), \mathbf{v} \in \mathbf{J}_{0, S}^{1}(\Omega)$, and $\nabla \tilde{p} \in \mathbf{G}_{h, S}(\Omega)$, then the following formula holds (see [KKN, p. 115], [K1]):

$$
\begin{aligned}
\int_{\Omega}( & \left.-\nu P_{0, S} \Delta \mathbf{u}+\frac{1}{\rho} \nabla \tilde{p}\right) \cdot \overline{\mathbf{v}} d \Omega \\
& =\nu E(\mathbf{u}, \mathbf{v})-\nu \int_{\Gamma} \sum_{i=1}^{2}\left(\frac{\partial u_{i}}{\partial x_{3}}+\frac{\partial u_{3}}{\partial x_{i}}\right) \overline{v_{i}} d \Gamma-\int_{\Gamma}\left(-\frac{1}{\rho} \tilde{p}+2 \nu \frac{\partial u_{3}}{\partial x_{3}}\right) \overline{v_{3}} d \Gamma
\end{aligned}
$$

where $E(\mathbf{u}, \mathbf{v})$ is the bilinear form corresponding to the quadratic form $E(\mathbf{u}, \mathbf{u})$.

The second auxiliary problem: S. G. Krein's Problem. Solve the following boundary value problem for the unknown functions $\mathbf{u} \in \mathbf{J}_{0, S}^{1}(\Omega)$ and $\nabla p_{2} \in$ $\mathbf{G}_{h, S}(\Omega)$ :

$$
\begin{aligned}
& -\nu P_{0, S} \Delta \mathbf{u}+\frac{1}{\rho} \nabla p_{2}=\mathbf{f}_{1}, \quad \operatorname{div} \mathbf{u}=0 \quad \text { in } \Omega, \\
& \mathbf{u}=0 \quad \text { on } S, \quad \nu\left(\frac{\partial u_{i}}{\partial x_{3}}+\frac{\partial u_{3}}{\partial x_{i}}\right)=0 \quad \text { on } \Gamma, \quad i=1,2, \\
& -p_{2}+2 \rho \nu \frac{\partial u_{3}}{\partial x_{3}}=0 \quad \text { on } \Gamma .
\end{aligned}
$$

If $\mathbf{u} \in \mathbf{J}_{0, S}^{1}(\Omega) \cap \mathbf{H}^{2}(\Omega)$, then, by the Embedding Theorem (see [G]), we have $\partial u_{3} / \partial x_{3} \in H_{\Gamma}^{1 / 2}$. Thus, by the definition of $\mathbf{G}_{h, S}(\Omega)$ in (3.8) , if $\mathbf{u} \in \mathbf{J}_{0, S}^{1}(\Omega) \cap \mathbf{H}^{2}(\Omega)$ and $\nabla p_{2} \in \mathbf{G}_{h, S}(\Omega)$ are solutions of (3.24) - (3.26), then $p_{2}$ solves the Zaremba problem (3.20) with $\psi:=2 \rho \nu \partial u_{3} / \partial x_{3}$ and hence, by Lemma 3.1,

$$
\nabla p_{2}=2 \rho \nu G \frac{\partial u_{3}}{\partial x_{3}}=: \nu L \mathbf{u} .
$$

Therefore, in this case, (3.24) can be written in the form $\nu A_{0} \mathbf{u}=\mathbf{f}_{\mathbf{1}}, \mathbf{u} \in \mathcal{D}\left(A_{0}\right)$, where the linear operator $A_{0}$ in $\mathbf{J}_{0, S}(\Omega)$ is given by

$$
A_{0} \mathbf{u}:=-P_{0, S} \Delta \mathbf{u}+\frac{1}{\rho} L \mathbf{u}, \quad \mathcal{D}\left(A_{0}\right)=\mathbf{J}_{0, S}^{1}(\Omega) \cap \mathbf{H}^{2}(\Omega) .
$$

Definition 3.3. A function $\mathbf{u} \in \mathbf{J}_{0, S}^{1}(\Omega)$ is said to be a generalized solution of the second auxiliary problem (3.24) $-(\underline{3.26})$ if for any $\mathbf{v} \in \mathbf{J}_{0, S}^{1}(\Omega)$ we have

$$
\nu E(\mathbf{u}, \mathbf{v})=\left(\mathbf{f}_{1}, \mathbf{v}\right) .
$$


From Green's formula (3.23) it follows that any classical solution of the second auxiliary problem is a generalized solution.

Lemma 3.4. If $\mathbf{f}_{1} \in \mathbf{J}_{0, S}(\Omega)$, then problem (3.24) - (3.26) has a unique generalized solution $\mathbf{u}=\nu^{-1} A^{-1} \mathbf{f}_{1}$ where $A$ is the Friedrichs extension of the operator $A_{0}$ defined in (3.28). The operator $A$ acts in the space $\mathbf{J}_{0, S}(\Omega)$, it is selfadjoint and strictly positive and has the following properties:

i) $\mathcal{D}(A) \subset \mathcal{D}\left(A^{1 / 2}\right)=\mathbf{J}_{0, S}^{1}(\Omega) \subset \mathbf{J}_{0, S}(\Omega), \overline{\mathcal{D}(A)}=\mathbf{J}_{0, S}(\Omega)$.

ii) If $\mathbf{u} \in \mathcal{D}(A), \mathbf{v} \in \mathbf{J}_{0, S}^{1}(\Omega)$, then

$$
(A \mathbf{u}, \mathbf{v})=E(\mathbf{u}, \mathbf{v})
$$

If $\mathbf{u}, \mathbf{v} \in \mathbf{J}_{0, S}^{1}(\Omega)$, then

$$
E(\mathbf{u}, \mathbf{v})=\left(A^{1 / 2} \mathbf{u}, A^{1 / 2} \mathbf{v}\right) .
$$

iii) The inverse operator $A^{-1}$ acting in $\mathbf{J}_{0, S}(\Omega)$ is compact and positive.

iv) The operator $A$ has discrete positive spectrum $\left\{\lambda_{k}(A)\right\}_{k=1}^{\infty}$ with accumulation point $+\infty$ and with asymptotic behaviour

$$
\lambda_{k}(A)=\left(\frac{\operatorname{mes} \Omega}{3 \pi^{2}}\right)^{-2 / 3} k^{2 / 3}(1+\mathrm{o}(1)), \quad k \rightarrow \infty .
$$

Proof. See [KKN, pp. 277-279]. The asymptotic formula (3.30) was deduced by G. Metivier $[\mathrm{M}]$ for Dirichlet and Neumann boundary conditions. By means of variation principles it follows that it is true in our case as well.

3.5. Transition to a differential operator equation of second order. In this subsection we return to the initial boundary value problem (3.15) - (3.18) and suppose that it has a classical solution $\{\mathbf{w} ; \tilde{p}\}$. In the following we will represent $\tilde{p} \in H_{\Gamma}^{1}(\Omega)$ in the form $\tilde{p}=p_{1}+p_{2}$ with $p_{1}, p_{2} \in H_{\Gamma}^{1}(\Omega)$ so that

$$
\nabla \tilde{p}=\nabla p_{1}+\nabla p_{2}, \quad \nabla p_{i} \in \mathbf{G}_{h, S}(\Omega), \quad i=1,2,
$$

where $\nabla p_{1}$ is a solution of the first auxiliary problem for

$$
\psi:=\rho g \gamma_{n} \mathbf{w}
$$

and $\left\{\mathbf{u}=(\partial \mathbf{w} / \partial t) ; \nabla p_{2}\right\}$ is a solution of the second auxiliary problem for

$$
\mathbf{f}_{1}:=-\frac{1}{\rho} \nabla p_{1}-\frac{\partial^{2} \mathbf{w}}{\partial t^{2}}+P_{0, S} \mathbf{f} .
$$

Here the operator $\gamma_{n}: \mathbf{H}^{1}(\Omega) \rightarrow H_{\Gamma}^{1 / 2}$ is defined by

$$
\gamma_{n} \mathbf{u}:=(\mathbf{u} \cdot \mathbf{n})_{\Gamma}=\left(\mathbf{u} \cdot \mathbf{e}_{3}\right)_{\Gamma}, \quad \mathcal{D}\left(\gamma_{n}\right):=\mathbf{J}_{0, S}(\Omega) \cap \mathbf{H}^{1}(\Omega)
$$

with $\gamma_{n} \mathbf{u} \in H_{\Gamma}^{1 / 2}=\mathcal{D}(G)$ for $\mathbf{u} \in \mathcal{D}\left(\gamma_{n}\right)$ by the Trace Theorem, where $G$ is the operator defined in Lemma 3.1 .

For $p_{1}$ we have, by Lemma 3.1

$$
\nabla p_{1}=G \psi=\rho g G \gamma_{n} \mathbf{w},
$$

where $G: H_{\Gamma}^{1 / 2} \rightarrow \mathbf{G}_{0, S}(\Omega) \subset \mathbf{J}_{0, S}(\Omega)$ is a bounded linear operator. For $\mathbf{u}$ we have, by Lemma 3.4 and using (3.31), (3.33),

$$
\mathbf{u}=\frac{\partial \mathbf{w}}{\partial t}=\nu^{-1} A^{-1}\left(-g G \gamma_{n} \mathbf{w}-\frac{\partial^{2} \mathbf{w}}{\partial t^{2}}+P_{0, S} \mathbf{f}\right) .
$$


From this it follows that a classical solution $\mathbf{w}$ of the boundary value problem (3.1) - (3.4) (and, correspondingly, of the problem (3.13), (3.15) - (3.18) ) is a solution of the Cauchy problem

$$
\frac{d^{2} \mathbf{w}}{d t^{2}}+\nu A \frac{d \mathbf{w}}{d t}+g G \gamma_{n} \mathbf{w}=P_{0, S} \mathbf{f}, \quad \mathbf{w}(0)=\mathbf{w}^{0}, \quad \frac{d \mathbf{w}}{d t}(0)=\mathbf{w}^{1} .
$$

Here $\mathbf{w}$ is the unknown function with values in the space $\mathbf{J}_{0, S}(\Omega), \nu>0$ is the kinematic viscosity of the fluid, $g>0$ is the acceleration due to gravity, $\mathbf{f}$ is a given function (the field of external forces), the operator $A$ is the operator of the second auxiliary problem, the operator $G$ is the operator of the first auxiliary problem, and the operator $\gamma_{n}$ is defined as in (3.32).

Definition 3.5. A function $\mathbf{w}$ is called a strong solution (with respect to the variable $t$ ) of the boundary value problem (3.1) - (3.4) on the interval $[0, T]$ if the Cauchy problem (3.35) has a strong solution $\mathbf{w}$ on $[0, T]$, i.e., if $\mathbf{w} \in C^{2}\left[0, T ; \mathbf{J}_{0, S}(\Omega)\right], \partial \mathbf{w} / \partial t$ $\in \mathcal{D}(A), A \partial \mathbf{w} / \partial t \in C\left[0, T ; \mathbf{J}_{0, S}(\Omega)\right], \mathbf{w} \in \mathcal{D}\left(g G \gamma_{n}\right), g G \gamma_{n} \mathbf{w} \in C\left[0, T ; \mathbf{J}_{0, S}(\Omega)\right]$, the conditions $\operatorname{div} \mathbf{w}=0$ in $\Omega, \mathbf{w}=0$ on $S$ hold, and if $\mathbf{w}$ satisfies the differential equation in (3.35) on $[0, T]$ as well as the initial conditions in (3.35).

3.6. Test of hypotheses. In this subsection we show that the Cauchy problem (3.35) is a special case of the general Cauchy problem (2.1).

To this end, we consider the Hilbert space $\mathcal{H}:=\mathbf{J}_{0, S}(\Omega)$ and therein the operators

$$
F:=\nu A, \quad \mathcal{D}(F):=\mathcal{D}(A), \quad B:=g G \gamma_{n}, \quad \mathcal{D}(B):=\mathcal{D}\left(G \gamma_{n}\right), \quad K:=0 .
$$

Then equation (3.35) is of the form (2.1) with $K=0$. In the sequel, we are going to verify that the conditions (2.2), (2.3), and (2.4) are satisfied for the operators in (3.36).

First, by Lemma 3.4 the operator $A$ is selfadjoint and strictly positive. Since $\nu>0$ and $K=0$, the operators $F=\nu A$ and $K$ satisfy the conditions (2.2), (2.3). It remains to study the properties of the operator $B$.

Lemma 3.6. The following relations hold:

$$
\mathcal{D}\left(A^{1 / 2}\right) \subset \mathcal{D}\left(\gamma_{n}\right) \subset \mathcal{D}\left(G^{*}\right), \quad G^{*} \mid \mathcal{D}\left(\gamma_{n}\right)=\gamma_{n} .
$$

Proof. The first inclusion follows from Lemma 3.4 by which $\mathcal{D}\left(A^{1 / 2}\right)=\mathbf{J}_{0, S}^{1}(\Omega) \subset$ $\mathbf{J}_{0, S}(\Omega) \cap \mathbf{H}^{1}(\Omega)=\mathcal{D}\left(\gamma_{n}\right)$.

Now let $\mathbf{v} \in \mathcal{D}\left(\gamma_{n}\right)=\mathbf{J}_{0, S}(\Omega) \cap \mathbf{H}^{1}(\Omega), \psi \in H_{\Gamma}^{1 / 2}$. Then, by Lemma 3.1. $\nabla p_{1}=$ $G \psi \in \mathbf{G}_{h, S}(\Omega)$ and $\gamma_{n} \mathbf{v} \in H_{\Gamma}^{1 / 2}$. Observing that $\operatorname{div} \mathbf{v}=0$ in $\Omega$, we see

$$
\begin{aligned}
\left(\nabla p_{1}, \mathbf{v}\right) & =\int_{\Omega} \nabla p_{1} \cdot \overline{\mathbf{v}} d \Omega=\int_{\Omega} \operatorname{div}\left(p_{1} \overline{\mathbf{v}}\right) d \Omega \\
& =\int_{\partial \Omega} p_{1} \overline{\mathbf{v} \cdot \mathbf{n}} d S=\int_{\Gamma} p_{1} \overline{\gamma_{n} \mathbf{v}} d \Gamma=\left(p_{1}, \gamma_{n} \mathbf{v}\right)_{0},
\end{aligned}
$$

that is,

$$
(G \psi, \mathbf{v})=\left(\psi, \gamma_{n} \mathbf{v}\right)_{0}, \quad \psi \in H_{\Gamma}^{1 / 2}, \mathbf{v} \in \mathcal{D}\left(\gamma_{n}\right)=\mathbf{J}_{0, S}(\Omega) \cap \mathbf{H}^{1}(\Omega) .
$$

This shows that if $G$ is considered as an operator acting from $\mathbf{L}_{2}(\Omega)$ to $L_{2}(\Gamma)$, then $\mathcal{D}\left(\gamma_{n}\right) \subset \mathcal{D}\left(G^{*}\right)$ and $G^{*} \mid \mathcal{D}\left(\gamma_{n}\right)=\gamma_{n}$.

Consider now the operator $G \gamma_{n}$ in $\mathbf{J}_{0, S}(\Omega)$ with dense domain

$$
\mathcal{D}\left(G \gamma_{n}\right):=\mathbf{J}_{0, S}(\Omega) \cap \mathbf{H}^{1}(\Omega)=\mathcal{D}\left(\gamma_{n}\right) .
$$


Theorem 3.7. The operator $G \gamma_{n}$ in $\mathbf{J}_{0, S}(\Omega)$ defined on the domain (3.39) is an unbounded symmetric nonnegative operator. Its Friedrichs extension $\widetilde{G \gamma_{n}}=G \widetilde{\gamma}_{n}$ which is defined on the domain

$$
\mathcal{D}\left(\widetilde{G \gamma_{n}}\right)=\mathbf{J}_{0}(\Omega) \oplus \mathbf{G}_{h, S}^{1}(\Omega),
$$

where

$$
\mathbf{G}_{h, S}^{1}(\Omega):=\left\{\mathbf{u} \in \mathbf{G}_{h, S}(\Omega): \mathbf{u}=\nabla \varphi, \widetilde{\gamma}_{n} \mathbf{u}:=\left(\frac{\partial \varphi}{\partial n}\right)_{\Gamma} \in H_{\Gamma}^{1 / 2}\right\},
$$

is an unbounded selfadjoint nonnegative operator with kernel

$$
\operatorname{Ker} \widetilde{G \gamma_{n}}=\mathbf{J}_{0}(\Omega) \text {. }
$$

On the space $\mathbf{G}_{h, S}^{1}(\Omega)$ the operator $\widetilde{G \gamma_{n}}$ is strictly positive, it has discrete spectrum $\left\{\lambda_{k}\right\}_{k=1}^{\infty}, 0<\lambda_{1} \leq \lambda_{2} \leq \ldots \leq \lambda_{k} \leq \ldots, \lambda_{k} \rightarrow \infty, k \rightarrow \infty$, its eigenvectors $\left\{\nabla \varphi_{k}\right\}_{k=1}^{\infty}$ form an orthogonal basis in the space $\mathbf{G}_{h, S}(\Omega)$,

$$
\left(\left(\frac{\partial \varphi_{k}}{\partial n}\right)_{\Gamma},\left(\frac{\partial \varphi_{l}}{\partial n}\right)_{\Gamma}\right)_{0}=\lambda_{k}\left(\nabla \varphi_{k}, \nabla \varphi_{l}\right)=\lambda_{k} \delta_{k l}
$$

and the eigenvalues $\left\{\lambda_{k}\right\}_{k=1}^{\infty}$ have the asymptotic behaviour

$$
\lambda_{k}=\left(\frac{\operatorname{mes} \Gamma}{4 \pi}\right)^{-1 / 2} k^{1 / 2}(1+\mathrm{o}(1)), \quad k \rightarrow \infty .
$$

Proof. Let $\mathbf{u}$ and $\mathbf{v}$ belong to $\mathcal{D}\left(G \gamma_{n}\right)$. Then $\psi=\gamma_{n} \mathbf{u} \in H_{\Gamma}^{1 / 2}$ and, by (3.38),

$$
\begin{aligned}
\left(G \gamma_{n} \mathbf{u}, \mathbf{v}\right)_{L_{2}(\Omega)} & =\left(\gamma_{n} \mathbf{u}, \gamma_{n} \mathbf{v}\right)_{0}=\overline{\left(\gamma_{n} \mathbf{v}, \gamma_{n} \mathbf{u}\right)_{0}} \\
& =\overline{\left(G \gamma_{n} \mathbf{v}, \mathbf{u}\right)_{L_{2}(\Omega)}}=\left(\mathbf{u}, G \gamma_{n} \mathbf{v}\right)_{L_{2}(\Omega)},
\end{aligned}
$$

i.e., $G \gamma_{n}$ is symmetric. From (3.44) with $\mathbf{v}=\mathbf{u}$ it follows that

$$
\left(G \gamma_{n} \mathbf{u}, \mathbf{u}\right)=\left\|\gamma_{n} \mathbf{u}\right\|_{0}^{2} \geq 0, \quad \mathbf{u} \in \mathcal{D}\left(G \gamma_{n}\right),
$$

i.e., the operator $G \gamma_{n}$ is nonnegative. Therefore it admits a nonnegative selfadjoint extension, the Friedrichs extension $\widetilde{G \gamma_{n}}$. It is evident from $(3.45)$ that

$$
\operatorname{Ker}\left(G \gamma_{n}\right)=\left\{\mathbf{u} \in \mathcal{D}\left(G \gamma_{n}\right)=\mathcal{D}\left(\gamma_{n}\right): \gamma_{n} \mathbf{u}=0\right\}=\operatorname{Ker}\left(\gamma_{n}\right)=\mathbf{J}_{0}(\Omega) \subset \mathbf{J}_{0, S}^{1}(\Omega)
$$

(see AHKM, Remark 3.7]). Since this set is dense in $\mathbf{J}_{0}(\Omega)$, then, after the extension,

$$
\operatorname{Ker} \widetilde{G \gamma_{n}}=\mathbf{J}_{0}(\Omega) \subset \mathbf{J}_{0, S}(\Omega) .
$$

According to the Spectral Theorem for selfadjoint operators, the orthogonal complement $\mathbf{G}_{h, S}(\Omega)=\mathbf{J}_{0, S}(\Omega) \ominus \mathbf{J}_{0}(\Omega)$ is an invariant subspace for the operator $\widetilde{G \gamma_{n}}$ and it corresponds to the positive part of its spectrum. Consider now the operator $\widetilde{G \gamma_{n}}$ on the subspace $\mathbf{G}_{h, S}(\Omega)$.

First, it follows from Lemma 3.1 and the Embedding Theorem (for the Lipschitz domain $\Omega$, see [G] that $G \psi \in \mathbf{G}_{h, S}(\Omega)$ if and only if $\psi \in H_{\Gamma}^{1 / 2}$. Hence, the operators $G: H_{\Gamma}^{1 / 2} \rightarrow \mathbf{G}_{h, S}(\Omega)$ and $G^{-1}: \mathbf{G}_{h, S}(\Omega) \rightarrow H_{\Gamma}^{1 / 2}$ are one-to-one. Therefore $\widetilde{G \gamma_{n}}=G \widetilde{\gamma}_{n}$ where $\widetilde{\gamma}_{n}$ is an extension of the operator $\gamma_{n}$ defined in (3.32) such that $\widetilde{\gamma}_{n}=0$ on $\mathbf{J}_{0}(\Omega)$ (see (3.46)) and $\widetilde{\gamma}_{n} \mathbf{u} \in H_{\Gamma}^{1 / 2}$ for $\mathbf{u}=\nabla \varphi \in \mathbf{G}_{h, S}(\Omega)$. From this the assertion (3.40) follows. 
Secondly, we consider the spectral problem for the operator $G \widetilde{\gamma}_{n}$ on $\mathbf{G}_{h, S}(\Omega)$ :

$$
G \widetilde{\gamma}_{n} \mathbf{u}=\lambda \mathbf{u}, \quad \mathbf{u}=\nabla \varphi \in \mathbf{G}_{h, S}^{1}(\Omega) .
$$

This problem can be rewritten in the form

$$
\begin{aligned}
& G\left(\frac{\partial \varphi}{\partial n}\right)_{\Gamma}=\lambda \nabla \varphi=\lambda G\left(\left.\varphi\right|_{\Gamma}\right), \\
& \Delta \varphi=0 \quad \text { in } \Omega, \quad \frac{\partial \varphi}{\partial n}=0 \quad \text { on } S, \quad \int_{\Gamma} \varphi d \Gamma=0 .
\end{aligned}
$$

From (3.47) it follows that

$$
\frac{\partial \varphi}{\partial n}=\lambda \varphi \quad \text { on } \Gamma .
$$

Problem (3.49), (3.48) is well known as Steklov's problem which arises in the study of oscillations of an ideal fluid in an open vessel (see, for instance, [KKN p. 140]). It can be reformulated as a spectral problem

$$
\psi=\lambda C \psi, \quad \psi=\left(\frac{\partial \varphi}{\partial n}\right)_{\Gamma} \in H=L_{2}(\Gamma) \ominus\left\{1_{\Gamma}\right\},
$$

where the operator $C$ is compact and positive. Therefore this problem has discrete spectrum, say $\left\{\lambda_{k}\right\}_{k=1}^{\infty}$, consisting of positive eigenvalues $\lambda_{k}$ with limit point $+\infty$, and the system of eigenvectors $\left\{\psi_{k}\right\}_{k=1}^{\infty}$ can be chosen to form an orthonormal basis in $H$. From this the basis property of the eigenvectors $\left\{\nabla \varphi_{k}\right\}_{k=1}^{\infty}, \nabla \varphi_{k}=$ $\lambda_{k}^{-1} G\left(\frac{\partial \varphi_{k}}{\partial n}\right)_{\Gamma}=\lambda_{k}^{-1} G \psi_{k}$, and the formulas (3.42) follow.

The asymptotic formula (3.43) can be derived by means of the variation ratio

$$
\int_{\Gamma}\left|\frac{\partial \varphi}{\partial n}\right|^{2} d \Gamma / \int_{\Omega}|\nabla \varphi|^{2} d \Omega
$$

or by means of the variation ratio

$$
\int_{\Omega}|\nabla \varphi|^{2} d \Omega / \int_{\Gamma}|\varphi|^{2} d \Gamma
$$

both of which have to be considered on the set of functions $\varphi$ satisfying (3.48) (see SV] $)$.

Corollary 3.8. The operator coefficients $F=\nu A, B=g G \gamma_{n}$, and $K=0$ of the Cauchy problem (3.35) satisfy the assumption (2.31) of Theorem 2.7: $F \gg 0, B \geq 0$ with $\mathcal{D}(B) \supset \mathcal{D}(F)$, and $K=0$.

Proof. It remains to prove (2.4). From (3.37), (3.39), and (3.40) it follows that

$$
\mathcal{D}(A) \subset \mathcal{D}\left(A^{1 / 2}\right) \subset \mathcal{D}\left(\gamma_{n}\right)=\mathcal{D}\left(G \gamma_{n}\right)=\mathcal{D}(B) \subset \mathcal{D}\left(G \widetilde{\gamma}_{n}\right)=\mathcal{D}(\widetilde{B})
$$

where $\widetilde{B}:=g G \widetilde{\gamma}_{n}$ is a nonnegative selfadjoint operator which is the Friedrichs extension of the operator $B=g G \gamma_{n}$. 
3.7. Theorem on well-posedness. According to the results of the last subsection, we can now apply Theorem 2.7] to the Cauchy problem (3.35) and obtain results for the original initial boundary value problem (3.1) - (3.4).

Theorem 3.9. Suppose that the following conditions hold for initial boundary value problem (3.35):

$$
\begin{aligned}
& \mathbf{w}^{0}=\mathbf{w}_{0}^{0}+\nabla \varphi^{0} \quad \text { with } \quad \mathbf{w}_{0}^{0} \in \mathbf{J}_{0}(\Omega), \nabla \varphi^{0} \in \mathbf{G}_{h, S}^{1}(\Omega), \\
& \mathbf{w}^{1} \in \mathcal{D}(A) \subset \mathcal{D}\left(A^{1 / 2}\right)=\mathbf{J}_{0, S}^{1}(\Omega), \\
& \mathbf{f} \in C^{1}\left[0, T ; \mathbf{L}_{2}(\Omega)\right] .
\end{aligned}
$$

Then the Cauchy problem (3.35) has a unique strong solution $\mathbf{w}$, that is, $\mathbf{w} \in$ $C^{2}\left[0, T ; \mathbf{J}_{0, S}(\Omega)\right], \partial \mathbf{w} / \partial t \in \mathcal{D}(A), A \partial \mathbf{w} / \partial t \in C\left[0, T ; \mathbf{J}_{0, S}(\Omega)\right], \mathbf{w} \in \mathcal{D}\left(g G \widetilde{\gamma}_{n}\right)$, as well as $g G \widetilde{\gamma}_{n} \mathbf{w} \in C\left[0, T ; \mathbf{G}_{h, S}(\Omega)\right]$, and equation (3.35) holds with the operator $\widetilde{\gamma}_{n}$ instead of $\gamma_{n}$.

If $\mathbf{w}^{\mathbf{0}} \in \mathcal{D}(A)$, then $\mathbf{w} \in \mathcal{D}(A)$ and $A \mathbf{w} \in C\left[0, T ; \mathbf{J}_{0, S}(\Omega)\right]$.

Proof. First, if $\mathbf{f} \in C^{1}\left[0, T ; \mathbf{L}_{2}(\Omega)\right]$, then $P_{0, S} \mathbf{f} \in C^{1}\left[0, T ; \mathbf{J}_{0, S}(\Omega)\right]$. Further, if $\mathbf{w}^{1} \in \mathcal{D}(A)$, then $\mathbf{w}^{1} \in \mathcal{D}(\nu A)=\mathcal{D}(F)$ (see (3.36)). At last, if the conditions on $\mathbf{w}^{0}$ in (3.50) hold, then, by Theorem 3.7, (3.40), (3.41), and Corollary [3.8, we have $\mathbf{w}^{0} \in \mathcal{D}\left(\widetilde{G \gamma_{n}}\right)=\mathcal{D}\left(G \widetilde{\gamma}_{n}\right)=\mathcal{D}(\widetilde{B})$ and $\mathcal{D}(F) \subset \mathcal{D}(\widetilde{B})$.

Therefore all conditions of Theorem 2.7 are satisfied for the Cauchy problem (3.35) and it has a unique strong solution $\mathbf{w}$ on the interval $[0, T]$ if we replace $\gamma_{n}$ by its extension $\widetilde{\gamma}_{n}$ to the space $\mathbf{J}_{0}(\Omega) \oplus \mathbf{G}_{h, S}^{1}(\Omega)$ (see (3.40) ). The last assertion follows by applying Remark 2.14 to the Cauchy problem (3.35).

As a corollary of Theorem 3.9 we obtain the following final result on the solvability of the initial boundary value problem (3.1) - (3.4).

Theorem 3.10. Let $A$ be the Friedrichs extension of the operator $A_{0}$ of the second auxiliary problem defined in (3.28) and suppose that the conditions

$$
\mathbf{w}^{0} \in \mathcal{D}\left(A^{1 / 2}\right), \quad \mathbf{w}^{1} \in \mathcal{D}(A), \quad \mathbf{f} \in C^{1}\left[0, T ; \mathbf{L}_{2}(\Omega)\right]
$$

hold. Then the problem (3.1) - (3.4) has a unique strong solution (with respect to the variable $t$ ) in the sense of Definition 3.5.

Proof. If the conditions (3.51) hold, then the conditions (3.50) of Theorem 3.9 are satisfied and, thus, the Cauchy problem (3.35) has a unique strong solution w on the interval $[0, T]$. Further, from the relation (2.78) we see that $A\left(\mathbf{w}-\mathbf{w}^{0}\right) \in C[0, T ; \mathcal{H}]$, i.e., $\mathbf{w}-\mathbf{w}^{0} \in C[0, T ; \mathcal{D}(A)]$. Since the operator $A^{-1 / 2}$ is bounded in $\mathbf{J}_{0, S}(\Omega)$ and $\mathbf{w}^{0} \in \mathcal{D}\left(A^{1 / 2}\right)$ by assumption (3.51), we conclude that the functions $\mathbf{w}-\mathbf{w}^{0}$ as well as w belong to $C\left[0, T ; \mathcal{D}\left(A^{1 / 2}\right)\right]=C\left[0, T ; \mathbf{J}_{0, S}^{1}(\Omega)\right]$. Therefore, by (3.37) and (3.39), we have $\mathbf{w}(t) \in \mathcal{D}\left(g G \gamma_{n}\right)$ for any $t \in[0, T]$ and $g G \tilde{\gamma}_{n} \mathbf{w}=g G \gamma_{n} \mathbf{w} \in C\left[0, T ; \mathbf{J}_{0, S}(\Omega)\right]$. Besides, since $\mathbf{w}(t) \in \mathbf{J}_{0, S}^{1}(\Omega)$ for any $t \in[0, T]$, we have $\operatorname{div} \mathbf{w}(t)=0$ in $\Omega$ and $\mathbf{w}(t)=0$ on $S$ for any $t \in[0, T]$ by definition of the space $\mathbf{J}_{0, S}^{1}(\Omega)$ (see (3.21)).

3.8. Oscillations of an ideal fluid in an open vessel. Consider now the degenerate case $\nu=0$ when the fluid in the vessel is inviscid (ideal). Then with the problem (3.1) - (3.4) we associate the Cauchy problem (3.35) with $\nu=0$, which is a classical Cauchy problem for a hyperbolic equation with operator coefficient 
$\widetilde{B}=g G \widetilde{\gamma}_{n}=\widetilde{B}^{*} \geq 0:$

$$
\frac{d^{2} \mathbf{w}}{d t^{2}}+\widetilde{B} \mathbf{w}=P_{0, S} \mathbf{f}, \quad \mathbf{w}(0)=\mathbf{w}^{0}, \quad \frac{d \mathbf{w}}{d t}(0)=\mathbf{w}^{1} .
$$

So, in this case, equation (3.13) takes the form

$$
\nabla \varphi=\rho P_{0, \Gamma} \mathbf{f},
$$

i.e., the projection $\nabla \varphi$ of the pressure $\nabla p$ on the subspace $\mathbf{G}_{0, \Gamma}(\Omega)$ is determined only by the corresponding projection of the external field of mass forces $\mathbf{f}$ and does not depend on the displacement field $\mathbf{w}$.

We will now derive the explicit form of the solution of problem 3.52) using the eigenvalues and eigenfunctions of the operator $\widetilde{B}=g G \widetilde{\gamma}_{n}$ (see Theorem 3.7).

According to the decomposition (3.10), we represent a solution $\mathbf{w} \in \mathbf{J}_{0, S}(\Omega)$ of problem (3.52) in the form

$$
\mathbf{w}=\mathbf{v}+\nabla \Phi \quad \text { with } \quad \mathbf{v} \in \mathbf{J}_{0}(\Omega), \quad \nabla \Phi \in \mathbf{G}_{h, S}(\Omega)
$$

and recall that $\operatorname{Ker} \widetilde{B}=\mathbf{J}_{0}(\Omega)$ (see (3.46) ). Then, after applying the orthogonal projections $P_{0}$ and $P_{h, S}$ of $\mathbf{J}_{0, S}(\Omega)$ onto the subspaces $\mathbf{J}_{0}(\Omega)$ and $\mathbf{G}_{h, S}(\Omega)$, respectively, to (3.52), we obtain the following two Cauchy problems:

$$
\begin{aligned}
& \frac{d^{2} \mathbf{v}}{d t^{2}}=P_{0} \mathbf{f}, \quad \mathbf{v}(0)=P_{0} \mathbf{w}^{0}, \quad \frac{d \mathbf{v}}{d t}(0)=P_{0} \mathbf{w}^{1}, \\
& \frac{d^{2} \nabla \Phi}{d t^{2}}+\widehat{B} \nabla \Phi=P_{h, S} \mathbf{f}, \\
& \nabla \Phi(0)=P_{h, S} \mathbf{w}^{0}=: \nabla \Phi^{0}, \quad \frac{d \nabla \Phi}{d t}(0)=P_{h, S} \mathbf{w}^{1}=: \nabla \Phi^{1},
\end{aligned}
$$

where

$$
\widehat{B}:=\widetilde{B} \mid \mathbf{G}_{h, S}^{1}(\Omega) .
$$

By Theorem 3.7, the operator $\widehat{B}$ is an unbounded positive definite operator which has discrete spectrum $\left\{\lambda_{k}(\widehat{B})\right\}_{k=1}^{\infty}, \lambda_{k}(\widehat{B})=g \lambda_{k}$ with $\lambda_{k}$ as in Theorem 3.7 having the asymptotic behaviour (3.43). For the corresponding system of eigenfunctions $\left\{\nabla \varphi_{k}\right\}_{k=1}^{\infty}$ we have (see (3.42)

$$
g\left(\left(\frac{\partial \varphi_{k}}{\partial n}\right)_{\Gamma},\left(\frac{\partial \varphi_{l}}{\partial n}\right)_{\Gamma}\right)_{0}=g \lambda_{k}\left(\nabla \varphi_{k}, \nabla \varphi_{l}\right)=\lambda_{k}(\widehat{B}) \delta_{k l} .
$$

Theorem 3.11. Suppose that for the initial boundary value problem (3.1) - (3.4) we have $\nu=0$ and the following conditions hold:

$$
\begin{array}{rrr}
\mathbf{f}=\mathbf{f}_{0}+\mathbf{f}_{0, \Gamma}+\nabla F \text { with } \quad \mathbf{f}_{0} \in C\left[0, T ; \mathbf{J}_{0}(\Omega)\right], \mathbf{f}_{0, \Gamma} \in C\left[0, T ; \mathbf{G}_{0, \Gamma}(\Omega)\right], \\
& \nabla F \in C^{1}\left[0, T ; \mathbf{G}_{h, S}(\Omega)\right], \\
\mathbf{w}^{0}=\mathbf{w}_{0}^{0}+\nabla \Phi^{0} \quad \text { with } \quad \mathbf{w}_{0}^{0} \in \mathbf{J}_{0}(\Omega), & \nabla \Phi^{0} \in \mathcal{D}(\widehat{B}) \subset \mathbf{G}_{h, S}(\Omega), \\
\mathbf{w}^{1}=\mathbf{w}_{0}^{1}+\nabla \Phi^{1} & \text { with } \quad \mathbf{w}_{0}^{1} \in \mathbf{J}_{0}(\Omega), & \nabla \Phi^{1} \in \mathcal{D}\left(\widehat{B}^{1 / 2}\right) \subset \mathbf{G}_{h, S}(\Omega) .
\end{array}
$$

Then the initial boundary value problem (3.1) - (3.4) has a unique strong solution, i.e., there exists a unique pair of functions $\{\mathbf{w} ; \nabla p\}$ such that

$(3.61) \mathbf{w}=\mathbf{v}+\nabla \Phi, \mathbf{v} \in C^{2}\left[0, T ; \mathbf{J}_{0}(\Omega)\right], \quad \nabla \Phi \in C[0, T ; \mathcal{D}(\widehat{B})] \cap C^{2}\left[0, T ; \mathbf{G}_{h, S}(\Omega)\right]$,

(3.62) $\nabla p=\nabla \varphi+\nabla \hat{p}, \nabla \varphi \in C\left[0, T ; \mathbf{G}_{0, \Gamma}(\Omega)\right], \nabla \hat{p}=\rho \widehat{B} \mathbf{w} \in C\left[0, T ; \mathbf{G}_{h, S}(\Omega)\right]$, 
and the relations (3.53) - (3.56) hold. In addition, we have

$$
\mathbf{v}(t)=\int_{0}^{t}(t-s) P_{0} \mathbf{f}(s) d s+\left(P_{0} \mathbf{w}^{1}\right) t+P_{0} \mathbf{w}^{0},
$$

and

$$
\begin{aligned}
\nabla \Phi(t) & =\cos \left(t \widehat{B}^{1 / 2}\right) \nabla \Phi^{0}+\widehat{B}^{-1 / 2}\left(\sin \left(t \widehat{B}^{1 / 2}\right) \nabla \Phi^{1}+\int_{0}^{t} \sin \left((t-s) \widehat{B}^{1 / 2}\right) \nabla F(s) d s\right) \\
& =\sum_{k=1}^{\infty} \nabla \varphi_{k}\left(\alpha_{k} \cos \left(\omega_{k} t\right)+\frac{1}{\omega_{k}} \beta_{k} \sin \left(\omega_{k} t\right)+\frac{1}{\omega_{k}} \int_{0}^{t} \sin \left(\omega_{k}(t-s)\right) f_{k}(s) d s\right)
\end{aligned}
$$

with $\omega_{k}:=\lambda_{k}(\widehat{B})^{1 / 2}, \alpha_{k}:=\left(\nabla \Phi^{0}, \nabla \varphi_{k}\right), \beta_{k}:=\left(\nabla \Phi^{1}, \nabla \varphi_{k}\right), f_{k}(t):=\left(\nabla F(t), \nabla \varphi_{k}\right)$, $k=1,2, \ldots$, where $\left\{\lambda_{k}(\widehat{B})\right\}_{k=1}^{\infty}$ and $\left\{\nabla \varphi_{k}\right\}_{k=1}^{\infty}$ are the eigenvalues and eigenfunctions of the operator $\widehat{B}$ (see (3.57)).

Proof. It follows from the above that the initial boundary value problem (3.1) (3.4) (in the case $\nu=0$ ) is equivalent to the relation (3.53) together with the two Cauchy problems (3.55) and (3.56). It is evident that problem (3.55) has a unique solution $\mathbf{v}$ of the form (3.63), and therefore under the assumptions (3.58) - (3.60) for $\mathbf{f}_{0}, \mathbf{w}^{0}$, and $\mathbf{w}^{1}$, we have $\mathbf{v} \in C^{2}\left[0, T ; \mathbf{J}_{0}(\Omega)\right]$. Further, since $\mathbf{f}_{0, \Gamma}=P_{0, \Gamma} \mathbf{f} \in$ $C\left[0, T ; \mathbf{G}_{0, \Gamma}(\Omega)\right]$, we see from 3.52 that $\nabla \varphi \in C\left[0, T ; \mathbf{G}_{0, \Gamma}(\Omega)\right]$. Moreover, it is known (see, e.g., $[\mathrm{BS}]$ ) that the inhomogeneous Cauchy problem (3.56) with unbounded positive definite operator coefficient $\widehat{B}=\widehat{B}^{*}$ has a unique strong solution of the form (3.64) if the conditions (3.58) - 3.60) for $\nabla F, \nabla \Phi^{0}$, and $\nabla \Phi^{1}$ hold. Finally, in the case $\nu=0$ (in contrast to the case $\nu>0$, see Subsection 3.5) we have $\nabla \tilde{p}=\nabla p_{1}\left(\right.$ since $\nabla p_{2}=0$ by (3.27) $)$, and therefore, by (3.33) and Theorem 3.7.

$$
\nabla \tilde{p}=\nabla p_{1}=\rho g G \widetilde{\gamma}_{n} \mathbf{w}=\rho \widehat{B} \mathbf{w} \in C\left[0, T ; \mathbf{G}_{h, S}(\Omega)\right] .
$$

As a corollary of Theorem 3.11 we obtain the following result.

Theorem 3.12. Under the conditions of Theorem 3.11 the law of full energy conservation holds for the strong solution of the initial boundary value problem (3.1) (3.4), which says that on the whole interval $[0, T]$,

$$
\begin{aligned}
\frac{1}{2}\left(\rho \int_{\Omega}\left|\frac{\partial \mathbf{w}}{\partial t}\right|^{2} d \Omega+\rho g \int_{\Gamma}\left|w_{n}\right|^{2} d \Gamma\right)= & \frac{1}{2}\left(\rho \int_{\Omega}\left|\mathbf{w}^{1}\right|^{2} d \Omega+\rho g \int_{\Gamma}\left|w_{n}^{0}\right|^{2} d \Gamma\right) \\
& +\rho \int_{0}^{t}\left(\int_{\Omega} \mathbf{f}(s) \cdot \frac{\partial \mathbf{w}}{\partial s}(s) d \Omega\right) d s .
\end{aligned}
$$

The proof is left to the reader.

Remark 3.13. The left-hand side in (3.65) is the total (kinetic plus potential) energy of the considered hydrodynamical system at time $t$ and the right-hand side is the sum of the total energy at initial time $t=0$ plus the work of the external forces on the system from time $t=0$ to time $t$.

Remark 3.14. Suppose that the condition

$$
\Delta \frac{\partial \mathbf{w}}{\partial t} \in C\left[0, T ; \mathbf{L}_{2}(\Omega)\right]
$$


is satisfied for problem (3.1) - (3.4) in the case $\nu>0$. Then under the assumptions of Theorem 3.9] the law of full energy conservation holds for the strong solution and it has the form (3.65) with the additional term

$$
-\rho \nu \int_{0}^{t} E\left(\frac{\partial \mathbf{w}}{\partial s}, \frac{\partial \mathbf{w}}{\partial s}\right) d s
$$

on the right-hand side of (3.65), where the form $E(\mathbf{u}, \mathbf{u})$ is defined by (3.22). The term (3.66) corresponds to the work of the internal dissipative forces in the hydrodynamical system. The proof of the law of energy conservation in this case is the same as in the case $\nu=0$.

Remark 3.15. From the physical point of view, the quantities $\omega_{k}$ in (3.64) are the frequencies of the eigenoscillations due to gravitation of an ideal fluid in an open vessel (the so-called surface waves).

\section{REFERENCES}

[ALMS] F. V. Atkinson, H. Langer, R. Mennicken, A. A. Shkalikov, The essential spectrum of some matrix operators, Math. Nachr. 67 (1994), 5-20. MR 1285306 (95f:47007)

[AdL] V.M. Adamyan, H. Langer, Spectral properties of a class of rational operator valued functions, J. Operator Theory 33 (1995), 259-277. MR 1354980 (96i:47023)

[AdLMS] V.M. Adamyan, H. Langer, R. Mennicken, J. Saurer, Spectral components of selfadjoint block operator matrices with unbounded entries, Math. Nachr. 178 (1996), 43-80. MR 1380703 (97i:47036)

[AKL] N. K. Askerov, S. G. Kreĭn, G. I. Laptev, Zadachi o kolebanijah viazkoy zhidkosti i sviazannije s ney operatornije uravnenija, Funktsional'niy analiz i ego prilozhenija, 2:2 (1968), 21-32 (in Russian). MR 0232233 (38:559)

[AHKM] T. Ya. Azizov, V. Hardt, N. D. Kopachevsky, R. Mennicken, To the problem on small motions and normal oscillations of a viscous fluid in a partially filled container, Math. Nachr. 248/249 (2003), 3-39. MR 1950713 (2003m:76046)

[AKO] T. Ya. Azizov, N. D. Kopachevsky, L. D. Orlova, Evoljutsionnije i speltral'nije zadachi, porozhdionnije problemoy malih dvizheniy viazkouprugoy zhidkosti, Trudy Sankt Peterburgskogo matematicheskogo obschestva. 6 (1998), 5-33 (in Russian).

[BS] M.Sh.Birman, M.Z. Solomjak, Spectral theory of selfadjoint operators in Hilbert space, Engl. transl.: Mathematics ad its Applications (Soviet Series), D. Reidel Publishing Co., Dordrecht, 1987. MR 1192782 (93g:47001)

[EE] D. E. Edmunds, W. D. Evans, Spectral theory and differential operators, Oxford Science Publications, The Clarendon Press, Oxford University Press, New York, 1987. MR 0929030 (89b:47001)

[En1] K.-J.Engel, Operator matrices and systems of evolution equations, RIMS Kokyuroku 966 (1996), 61-80. MR 1484209 (99a:34161)

[En2] K.-J.Engel, Matrix representation of linear operators on product spaces, in: Aiena, P. (ed.) et al., International workshop on Operator theory, Cefalu, Italy, July 14-19, 1997. Palermo: Circolo Matematico di Palermo, Suppl. Rend. Circ. Mat. Palermo, II. Ser. 56 (1998), 219-224. MR 1710840 (2000f:47004)

[F] H. O. Fattorini: Second Order Linear Differential Equations in Banach Spaces, NorthHolland Mathematics Studies 108, Notas de Matemática [Mathematical Notes] 99, North-Holland Publishing Co., Amsterdam, 1985. MR 0797071 (87b:34001)

[G] E. Gagliardo, Caratterizzazioni delle tracce sulla frontiera relative ad alcune classi di funzioni in $n$ variabili, Rend. Sem. Mat. Univ. Padova 27 (1957), 284-305 (in Italian). MR 21:1525

[Ga] A. Garadzhaev, K zadache o normal'nih kolebanijah tiazhioloy viazkoy zhidkosti v sosude, Sibirskiy matematicheskiy zhurnal 25:2 (1984), 213-216 (in Russian). MR 0741022 (86c:76025)

[Gob] J. Gobert, Une inégalité fondamentale de la théorie de l'élasticité, Bull. Soc. Roy. Sci. Liège 31 (1962), 182-191 (in French). MR 0133684 (24:A3510) 
[GGK] I. C. Gohberg, S. Goldberg, M. A. Kaashoek, Classes of Linear Operators, Vol. I, Operator Theory: Adv. Appl. 49, Birkhäuser Verlag, Basel, 1990. MR 1130394 (93d:47002)

[Go] D. Goldstein, Polugruppi lineynih operatorov i ih prilozhenija, Vischa shkola, Kiev, 1989 (in Russian). MR 1201586 (93i:47054)

[GG] V.I. Gorbachuk, M. L. Gorbachuk, Granichnye zadachi dlya differentsial'no-operator nykh uravneniı̌, Naukova Dumka, Kiev, 1984 (in Russian), Engl Transl. Boundary Value Problems for Operator Differential Equations, Kluwer Academic Publishers Group, Dordrecht, 1991. MR 1190695 (93e:00017)

[HMN] V. Hardt, R. Mennicken, S. Naboko, Systems of singular differential operators of mixed order and applications to 1-dimensional MHD problems, Math. Nachr. 205 (1999), 19-68. MR 1709162 (2000f:47073)

[HP] E. Hille, R. S. Phillips, Functional Analysis and Semi-Groups, American Mathematical Society Colloquium Publications 31, American Mathematical Society, Providence, R.I., 1957. MR 0089373 (19,664d)

[IMF] V. K. Ivanov, I. V. Mel'nikova, A. I. Filinkov, Operator-Differential Equations and IllPosed Problems, Moscow, Nauka, 1995 (in Russian). MR 1415388 (97j:47092)

[Ka] T. Kato, Perturbation theory for linear operators, 2nd edition, Springer Verlag, Berlin, Heidelberg, New York, 1995. MR 1335452 (96a:47025)

[Kon] A. Yu. Konstantinov, Spectral theory of some matrix differential operators of mixed order. Ukrăn. Mat. Zh. 50:8 (1998), 1064-1072 (in Ukrainian), Engl. Transl. Ukrainian Math. J. 50:8 (1998), 1212-1223 (1999). MR 1706515 (2001a:47047)

[Ko] N. D. Kopachevsky, Normal'nije kolebanija sistemy tiazhiolih viazkih vraschajuschihsia zhidkostey, Doklady AN Ukrainskoy SSR, serija A, 7 (1978), 586-590 (in Ukrainian).

[KKN] N. D. Kopachevsky, S. G. Kreĭn, Ngo Zuy Can, Operatornije metody v lineynoy gidrodinamike: Evoljutsionnije i spektral'nije zadachi [Operator Methods in Linear Hydrodynamics], Nauka, Moscow, 1989 (in Russian). MR 1037258 (91h:76001)

[KKMRSZ] A. I. Koshelev, M. A. Krasnosel'sky, S. G. Mihlin, L. S. Rakovschik, V. Ja. Stetsenko, P. P. Zabrejko, Integral'nije uravnenija, Nauka, Moscow, 1968 (in Russian).

[KZPP] M. A. Krasnosel'sky, P. P. Zabrejko, E. I. Pustyl'nik, P. E. Sobolevsky, Integral'nije operatori v prostranstvah summiruemih funktsiy, Nauka, Moscow, 1966 (in Russian). Engl. Transl.: Integral Operators in Spaces of Summable Functions, Monographs and Textbooks on Mechanics of Solids and Fluids, Mechanics: Analysis, Noordhoff International Publishing, Leiden, 1976.

[K1] S. G. Krel̆n, O kolebanijah viazkoy zhidkosti v sosude, Doklady AN SSSR 159:2 (1964), 262-265 (in Russian). MR 0182238 (31:6461)

[K2] S. G. Kreĭn, Linejnije differentsial'nije uravnenija v banahovom prostranstve, Nauka, Moscow, 1967 (in Russian), Engl. Transl.: Linear Differential Equations in Banach Space, Transl. Math. Monographs 29, American Mathematical Society, Providence, R.I., 1971. MR 0247239 (40:508)

[KH] S. G. Kreĭn, M. I. Hazan, Differentsial'nije uravnenija v banahovom prostranstve, V sbornike "Itogi nauki i tehniki", Matematicheskij analiz 21, 130-264 (in Russian).

[KL] S. G. Kreı̆n, G. I. Laptev, K zadache o dvizhenii viazkoy zhidkosti v otkritom sosyde, Functsional'niy analiz i ego prilozhenija 2:2 (1968), 40-50 (in Russian). MR $0248462(40: 1714)$

[La] O.A. Ladyzhenskaya, The Mathematical Theory of Viscous Incompressible Flow, Nauka, Moscow, 1970 (in Russian), Engl. Transl.: Mathematics and its Applications 2, Gordon and Breach, Science Publishers, New York, London, Paris, 1969. MR 0254401 (40:7610)

[LT1] H. Langer, C. Tretter, Spectral decomposition of some nonselfadjoint block operator matrices, J. Operator Theory 39:2 (1998), 339-359. MR 1620503 (99d:47004)

[LT2] H. Langer, C. Tretter, Diagonalization of certain block operator matrices and applications to Dirac operators, Operator Theory: Adv. Appl. 122 (2001), 331-358. MR 1846064 (2002f:47006)

$[\mathrm{MM}] \quad$ R. Mennicken, A. Motovilov, Operator interpretation of resonances arising in spectral problems for $2 \times 2$ operator matrices, Math. Nachr. 201 (1999), 117-181. MR 1680916 (2000f:47040) 
[MNT] R. Mennicken, S. Naboko, C. Tretter, Essential spectrum of a system of singular differential operators and the asymptotic Hain-Lüst operator, Proc. Amer. Math. Soc. 130 (2002), 1699-1710. MR 1887017 (2002k:47092)

[MS] R. Mennicken, A. A. Shkalikov, Spectral decomposition of symmetric operator matrices, Math. Nachr. 179 (1996), 259-273. MR 1389460 (97d:47012)

[M] G. Metivier, Valeurs propres d'opérateurs définis par la restriction de systèmes variationnels à des sous-espaces, J. Math. Pures Appl. (9) 57:2 (1978), 33-156 (in French). MR 0505900 (81c:35100)

[M] A. Motovilov, Removal of the resolvent-like energy dependence from interactions and invariant subspaces of a total Hamiltonian, J. Math. Phys. 36:12 (1995), 6647-6664. MR 1359649 (96m:81249)

[N1] R. Nagel, Towards a "matrix theory" for unbounded operator matrices, Math. Z. 201:1 (1989), 57-68. MR 0990188 (90c:47004)

[N2] R. Nagel, Operator matrices and reaction-diffusion systems, Rend. Sem. Mat. Fis. Milano 59 (1989), 185-196. MR 1159696 (93c:47085)

[N3] R. Nagel, The spectrum of unbounded operator matrices with nondiagonal domain, J. Funct. Anal. 89:2 (1990), 291-302. MR 1042212 (91b:47005)

[S] A. A. Shkalikov, On the essential spectrum of matrix operators, Mat. Zametki 58:6 (1995), 945-949 (in Russian). MR 1382107 (97e:47004)

[SV] I. L. Vulis, M.Z. Solomjak, Spectral asymptotic analysis of the degenerate Steklov problem, Vestnik Leningrad. Univ. No. 19 Mat. Meh. Astronom Vyp. 4 (1973), 148150, 156 (in Russian). MR 0330795 (48:9132)

[S1] M. Sowa, Cosine operator functions, Rozprawy Math. 49 (1966), 1-47. MR 0193525 (33:1745)

[S2] M. Sowa, Semigroups and cosine functions of normal operators in Hilbert spaces, Casopis Pest. Math. 93 (1968), 437-458. MR 0250121 (40:3361)

[T] R. Temam, Navier-Stokes Equations, Theory and Numerical Analysis, Studies in Mathematics and its Applications, 2. North-Holland Publishing Co., Amsterdam, 1979. MR 0603444 (82b:35133)

Taurida National V. Vernadsky University, Ul. Yaltinskaya, 4, 95007 Simferopol, CRimea, Ukraine

E-mail address: kopachevsky@tnu.crimea.ua

NWF I - Mathematik, University of Regensburg, 93040 Regensburg, Germany

E-mail address: reinhard.mennicken@mathematik.uni-regensburg.de

Taurida National V. Vernadsky University, Ul. Yaltinskaya, 4, 95007 Simferopol, Crimea, Ukraine

E-mail address: kromsh@crimea.com

FB 3 - Mathematik, University of Bremen, Bibliothekstr. 1, 28359 Bremen, Germany

E-mail address: ctretter@math.uni-bremen.de 